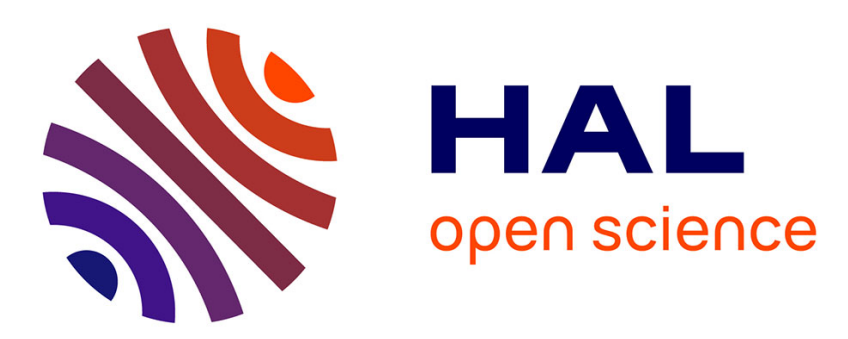

\title{
Extending the loop language with higher-order procedural variables
}

\author{
Tristan Crolard, Emmanuel Polonowski, Pierre Valarcher
}

\section{To cite this version:}

Tristan Crolard, Emmanuel Polonowski, Pierre Valarcher. Extending the loop language with higherorder procedural variables. ACM Transactions on Computational Logic, 2009, 10 (4), pp.1-37. 10.1145/1555746.1555750 . hal-00422158

\section{HAL Id: hal-00422158 https://hal.science/hal-00422158}

Submitted on 6 Oct 2009

HAL is a multi-disciplinary open access archive for the deposit and dissemination of scientific research documents, whether they are published or not. The documents may come from teaching and research institutions in France or abroad, or from public or private research centers.
L'archive ouverte pluridisciplinaire HAL, est destinée au dépôt et à la diffusion de documents scientifiques de niveau recherche, publiés ou non, émanant des établissements d'enseignement et de recherche français ou étrangers, des laboratoires publics ou privés. 


\title{
Extending the Loop Language with Higher-Order Procedural Variables
}

\author{
BY T. CROLARD, E. POLONOWSKI AND P. VALARCHER
}

Paris East University

March 22, 2008

\begin{abstract}
We extend Meyer and Ritchie's Loop language with higher-order procedures and procedural variables and we show that the resulting programming language (called Loop $\left.^{\omega}\right)$ is a natural imperative counterpart of Gödel System T. The argument is two-fold:

1. we define a translation of the $\mathrm{Loop}^{\omega}$ language into System $\mathrm{T}$ and we prove that this translation actually provides a lock-step simulation,

2. using a converse translation, we show that Loop ${ }^{\omega}$ is expressive enough to encode any term of System T.

Moreover, we define the "iteration rank" of a Loop ${ }^{\omega}$ program, which corresponds to the classical notion of "recursion rank" in System T, and we show that both translations preserve ranks. Two applications of these results in the area of implicit complexity are described.
\end{abstract}

\section{Introduction}

Primitive recursive functionals of finite type are representable as terms of the simply typed $\lambda$-calculus equipped with a type of natural numbers and primitive recursion at all types. This calculus (called System T) was first introduced by Gödel in its proof-theoretic study of Peano arithmetic (see its Dialectica paper on the consistency of arithmetic [Gödel, 1958], reproduced with English translation in [Gödel, 1990]). Moreover, System $\mathrm{T}$ is well suited to give a formal semantics to constructs found in (higher-order) programming languages [Girard et al., 1989].

The Loop language [Meyer and Ritchie, 1976] is a core imperative language in which programs consist only of assignments, sequences, and bounded loops. Meyer and Ritchie's proved in particular that LoOP programs compute exactly the class of primitive recursive functions. The Loop language has since been widely studied in the literature (see for instance the textbooks [Davis and Weyuker, 1983] and [Calude, 1988]).

In this paper, we introduce a statically typed extension of the Loop language (called Loop $^{\omega}$ ) with higher-order procedures and procedural variables and we argue that this programming language is a natural imperative counterpart of Gödel System T. The argument is two-fold:

1. we define a translation of the Loop ${ }^{\omega}$ language into System $\mathrm{T}$ and we prove that this translation actually provides a lock-step simulation,

2. using a converse translation, we show that $\operatorname{LOOP}^{\omega}$ is expressive enough to encode any term of System T.

The first result is the main contribution of this paper: we prove that Loop ${ }^{\omega}$ is not only extensionally equivalent to System $\mathrm{T}$, but also intensionally. In order to develop this point, we need to give some details about the formal semantics of $\operatorname{LoOP}^{\omega}$. The overall design of the language follows mostly the principles advocated in [Schmidt, 1994]. The operational semantics of $\mathrm{LOOP}^{\omega}$ is presented first as a natural semantics (also called "bigstep" semantics) in the style of [Kahn, 1987]. Then a transition semantics (also called "small-step" semantics [Plotkin, 1981]) tailored of the lock-step simulation and 
which refines the natural semantics is defined. The simulation theorem states that each evaluation step of a Loop ${ }^{\omega}$ program (according to the transition semantics) is mapped to one reduction step of the transformed program in System $\mathrm{T}$ (using a call-by-value strategy). As a corollary of this theorem, we obtain that all LoOP ${ }^{\omega}$ programs are always terminating.

Note that $\mathrm{LOOP}^{\omega}$ is a genuine imperative language with first-class procedures (true closures) and mutable procedural variables (aka function pointers). For instance, any LoOP ${ }^{\omega}$ can easily be written using $C$ \# syntax (where anonymous first-class procedures are called delegates [ISO, 2003]) and then compiled with a $C$ \# compiler. However, it is a "pure" imperative language in the following sense: its type system forbids side-effects, parameterinduced aliasing (which are controversial features known to complicate the semantics). Moreover the type system forbids the well-known back-patching technique which exploits procedural variables to define arbitrary recursive procedures [Landin, 1964] (and LoOP ${ }^{\omega}$ is thus a "total" programming language as advocated in [Turner, 2004]). As a consequence of these choices, both semantics presented in this paper are simple but rather unusual for imperative languages: they are both location-free semantics (see [Donahue, 1977], [Plotkin, 1981] and [Felleisen and Friedman, 1987]) and they rely crucially on the distinction between mutable and read-only (immutable) variables.

The second result is obtained by defining a converse translation (from System $\mathrm{T}$ into LoOP $^{\omega}$ ) and proving that the composition the two translations yields the identity in System T (up to $\beta \pi$-equivalence). Since both translations are syntax directed (compositional) and type-preserving, we can be even more specific about the correspondence between a functional program and its imperative counterpart. Recall that a major result concerning System T from [Kreisel, 1951] states that functions on the natural numbers that are definable in this system correspond exactly to functions that are provably total in first-order Peano arithmetic (see also the survey [Avigad and Feferman, 1998] or the textbook [Schütte, 1967]). More precisely, that there is a syntactic hierarchy of fragments $\mathrm{T}_{n}$ of System $\mathrm{T}$ such that the class of functions representable in $\mathrm{T}_{n}$ is identical to the class of functions provably recursive in the fragment of Peano arithmetic where induction is restricted to $\Sigma_{n+1}$ formulas. In particular, $\mathrm{T}_{0}$ corresponds to the class of primitive recursive functions.

We define thus a similar hierarchy of fragments LOOP $^{n}$ of LOOP $^{\omega}$ and we show that both translations relate programs of $\mathrm{LoOP}^{n}$ and terms of $\mathrm{T}_{n}$. As a corollary, we obtain that the functions representable in a language with higher-order procedures but without procedural variables (which is a sub-language of $\mathrm{LOOP}^{0}$ ) are primitive recursive. This corollary generalizes thus previous results presented in [Crolard et al., 2006] where Meyer and Ritchie's Loop language was translated into $\mathrm{T}_{0}$. On the other hand, the Ackermann function which is known not to be primitive recursive is representable in $\mathrm{T}_{1}$ and thus also in LOOP $^{1}$. As far as we know, LOOP ${ }^{\omega}$ is the first total imperative language allowing to program the Ackermann function.

Applications. A first application of these results is extensional: we derive a new characterization of the class of Csillag-Kalmar elementary functions (the class $\mathcal{E}_{3}$ in Grzegorczyk hierarchy). In [Beckmann and Weiermann, 2000], such a characterization is based on a syntactic restriction on terms of a variant of Gödel System T. As a corollary of various properties of our two translations we provide an imperative counterpart of this restriction. In particular, we obtain that any $\mathrm{LOOP}^{\omega}$ program in which any bound of loop is a readonly input variable is elementary.

A second application is intensional and is related to the so-called minimum problem. In [Colson and Fredholm, 1998], the authors proved that in call-by-value System T, any algorithm which computes a non-trivial binary function (where trivial means constant or projection plus constant), has a time-complexity which is at least linear in one of the inputs. As a consequence of this property, there is no term that computes the minimum of two natural numbers $n$ and $m$ in time $O(\min (n, m))$. As a corollary of the lock-step simulation we obtain a similar negative result for $\operatorname{Loop}^{\omega}$ programs. 
Related works. There has been a lot of work on the semantics of Algol (major contributions are collected in [O'Hearn and Tennent, 1997]) and this work has influenced the design of most modern programming languages. For instance, the Loop ${ }^{\omega}$ language is very close in spirit to the language partially described in [Reynolds, 1978] whose purpose was in particular to show that it is possible to avoid aliasing while retaining Algol-like higherorder procedures. More generally, our work can also be seen as providing a denotational semantics for an imperative language using a $\lambda$-calculus and thus follows the Scott-Strachey tradition [Stoy, 1977].

The idea of translating an imperative program into a functional one actually goes back to [McCarthy, 1960]. However, our translation is somewhat closer to the state monad [Moggi, 1991, Wadler, 1990] used to encode a mutable state (or references) in a pure functional language. Although this encoding is usually global and thus changes the type of all terms, a local encoding can be obtained if an effect system [Gifford and Lucassen, 1986] is used for the source language (instead of a conventional type system). A simulation based on such an encoding is described in [Wadler, 1998].

Similarly, in [Filliâtre, 2003], the author defines a monadic translation of simply-typed functional programs with references (annotated with Floyd-Hoare assertions) into a type theory. Their translation provides the core of the proof technique developed afterward for imperative programs [Filliâtre and Marché, 2004]. Although similar to the one described in this paper, their translation is only considered in the context of program verification (no simulation is defined).

Finally, compiling one programming language into another in order to derive complexity properties is a common approach. The reader is referred for instance to [Jones, 1997] for various applications of this technique.

Plan of the paper. In Section 2, we present our variant of Gödel System T. In Section 3, we describe the Loop ${ }^{\omega}$ language (syntax, type system and semantics). In Section 4, we show how to translate LOOP $^{\omega}$ programs into functional programs and we prove the simulation theorem. In Section 5, we define the converse translation, and we use it to prove that LoOP $^{\omega}$ is as expressive as System T. Finally, in Section 6, we describe the two applications.

\section{Gödel System T}

Gödel System $\mathrm{T}$ is usually defined as the simply typed $\lambda$-calculus extended with a type of natural numbers and with primitive recursion at all types. We consider in this paper a variant of System $\mathrm{T}$ with product types (tuples and $n$-ary functions) and a constant-time predecessor operation. Moreover, since we are mainly interested here in the call-by-value evaluation strategy, we formulate this system directly as a context semantics (a set of reduction rules together with an inductive definition of evaluation contexts). As usual, we consider terms up to $\alpha$-conversion and the set $F I d(t)$ of free identifiers of a term $t$ is defined in the standard way. The rewriting system is summarized in Figure 2.1, where variables $x, x_{1}, \ldots, x_{n}$ range over a set of identifiers and $t\left[v_{1} / x_{1}, \ldots, v_{n} / x_{n}\right]$ denotes the usual capture-avoiding substitution. We also recall the type system in Figure 2.2 and we consider only well-typed terms in the sequel.

Remark 2.1. In order to distinguish the successor $S$ (which is a constructor) from the successor seen as an operation (whose evaluation should imply a reduction step), we use the keyword succ as an abbreviation for $\lambda x . S(x)$. Note that we also consider a primitive constant-time predecessor (called pred) for complexity reasons: although this function is clearly definable in System $\mathrm{T}$ its complexity would be at least linear under the call-byvalue evaluation strategy [Colson and Fredholm, 1998]. More details on this question are given in Section 6.2 and Appendix A.

Notation 2.2. We use the more verbose syntax fn $(\vec{x}: \vec{\sigma}) \Rightarrow t$ instead of simply $\lambda \vec{x} . t$ whenever we wish to make the types explicit. Moreover, we write $\vec{\tau}^{\times}$as an abbreviation for $\tau_{1} \times \ldots \times \tau_{n}$ and we consider unit as the special case of $\vec{\tau} \times$ obtained when $n=0$. 
(types)

$$
\begin{aligned}
\tau::= & \text { int } \\
& \text { unit } \\
& \tau_{1} \rightarrow \tau_{2} \\
& \tau_{1} \times \ldots \times \tau_{n}
\end{aligned}
$$

(terms)

$$
\begin{aligned}
t::= & x \\
& \mid \\
& S(t) \\
& \operatorname{pred}(t) \\
& t_{1} t_{2} \\
& \lambda\left(x_{1}, \ldots, x_{n}\right) \cdot t \\
& \left(t_{1}, \ldots, t_{n}\right) \\
& \operatorname{rec}\left(t_{1}, t_{2}, t_{3}\right)
\end{aligned}
$$

(values)

$$
\begin{aligned}
v::= & x \\
\mid & 0 \\
\mid & S(v) \\
& \left(v_{1}, \ldots, v_{n}\right) \\
& \lambda\left(x_{1}, \ldots, x_{n}\right) . t
\end{aligned}
$$

(contexts)

$$
\begin{aligned}
& C[]::= {[] } \\
& C[] t \\
& v C[] \\
& S(C[]) \\
& \operatorname{pred}(C[]) \\
& \operatorname{rec}\left(C[], t_{2}, t_{3}\right) \\
& \mid \operatorname{rec}\left(v_{1}, C[], t_{3}\right) \\
& \operatorname{rec}\left(v_{1}, v_{2}, C[]\right) \\
&\left(v_{1}, \ldots v_{i-1}, C[], t_{i+1} \ldots, t_{n}\right)
\end{aligned}
$$

(evaluation rules)

$$
C[\operatorname{pred}(0)] \rightsquigarrow C[0]
$$$$
C[\operatorname{pred}(S(v)) \cdot \rightsquigarrow C \cdot v]
$$

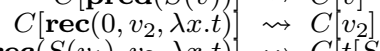

$C\left[\operatorname{rec}\left(S\left(v_{1}\right), v_{2}, \lambda x . t\right)\right] \rightsquigarrow C\left[t\left[S\left(v_{1}\right) / x\right] \operatorname{rec}\left(v_{1}, v_{2}, \lambda x . t\right)\right]$

$C\left[\lambda\left(x_{1}, \ldots, x_{n}\right) \cdot t\left(v_{1}, \ldots, v_{n}\right)\right] \rightsquigarrow C\left[t\left[v_{1} / x_{1}, \ldots, v_{n} / x_{n}\right]\right]$

Figure 2.1. Gödel System T

$$
\begin{gathered}
\frac{x: \tau \in \Gamma}{\Gamma \vdash x: \tau} \\
\Gamma \vdash 0: i n t \\
\frac{\Gamma \vdash t: i n t}{\Gamma \vdash S(t): i n t} \\
\frac{\Gamma \vdash t: i n t}{\Gamma \vdash \operatorname{pred}(t): i n t} \\
\frac{\Gamma \vdash t_{1}: \tau_{1} \quad \ldots \quad \Gamma \vdash t_{n}: \tau_{n}}{\Gamma \vdash\left(t_{1}, \ldots, t_{n}\right): \tau_{1} \times \ldots \times \tau_{n}} \\
\frac{\Gamma, x_{1}: \tau_{1}, \ldots, x_{n}: \tau_{n} \vdash t: \sigma}{\Gamma \vdash \lambda\left(x_{1}, \ldots, x_{n}\right) \cdot t:\left(\tau_{1} \times \ldots \times \tau_{n}\right) \rightarrow \sigma} \\
\frac{\Gamma \vdash t_{1}: \sigma \rightarrow \tau \quad \Gamma \vdash t_{2}: \sigma}{\Gamma \vdash t_{1} t_{2}: \tau} \\
\frac{\Gamma \vdash t_{1}: i n t \quad \text { (IDENT) }}{\Gamma \vdash t_{2}: \tau \quad \Gamma \vdash t_{3}: i n t \rightarrow \tau \rightarrow \tau} \\
\Gamma \vdash \mathbf{r e c}\left(t_{1}, t_{2}, t_{3}\right): \tau
\end{gathered}
$$

Figure 2.2. Functional type system

Remark 2.3. As usual [Landin, 1964], we write let $\left(x_{1}, \ldots, x_{n}\right)=u$ in $t$ be an abbreviation for the redex $\lambda\left(x_{1}, \ldots, x_{n}\right)$.t $u$. The following typing rule and evaluation rule can be obtained:

$$
\begin{gathered}
\frac{\Gamma \vdash u:\left(\tau_{1} \times \ldots \times \tau_{n}\right) \quad \Gamma, x_{1}: \tau_{1}, \ldots x_{n}: \tau_{n} \vdash t: \sigma}{\Gamma \vdash \operatorname{let}\left(x_{1}, \ldots, x_{n}\right)=u \text { in } t: \sigma} \\
C\left[\operatorname{let}\left(x_{1}, \ldots, x_{n}\right)=\left(v_{1}, \ldots, v_{n}\right) \text { in } t\right] \rightsquigarrow C\left[t\left[v_{1} / x_{1}, \ldots, v_{n} / x_{n}\right]\right]
\end{gathered}
$$


Finally, note that let $\left(x_{1}, \ldots, x_{n}\right)=[]$ in $t$ is an evaluation context.

Let us recall the well-known infinite syntactic hierarchy of fragment $T_{n}$ of Gödel System T. We call "recursion rank" of a term $t$ the maximum degree of the types of the recursors which occur in $t$, where the degree of a type is defined as follows:

Definition 2.4. The degree $\partial(\tau)$ of a type $\tau$ is defined inductively by:

$$
\begin{aligned}
& -\quad \partial(\text { int })=0 \\
& -\quad \partial(\sigma \rightarrow \tau)=\max (\partial(\sigma)+1, \partial(\tau)) \\
& -\quad \partial\left(\tau_{1} \times \ldots \times \tau_{n}\right)=\max \left(\partial\left(\tau_{1}\right), \ldots, \partial\left(\tau_{n}\right)\right)
\end{aligned}
$$

Remark 2.5. Using standard type isomorphisms, any type is isomorphic either to unit or to some type which does not contain unit. Thus we may consider without restriction that $\tau$ does not contain unit in the above definition.

Definition 2.6. The fragment $T_{n}$ of System $T$ is defined as the set of terms with recursion rank less or equal to $n$.

\subsection{Example: the Ackermann function}

Our running example in this paper is the Ackermann function. This function is known not to be primitive recursive [Peter, 1968] but it can be represented in System T, for instance as follows:

$$
a c k(m, n)=(\operatorname{rec}(m, \lambda y \cdot \operatorname{succ}(y), \lambda i \cdot \lambda h \cdot \lambda y \cdot \operatorname{rec}(y,(h S(0)), \lambda j \cdot \lambda k \cdot(h k))) n)
$$

It is well-known (see [Avigad and Feferman, 1998] for instance) that functions of type $N^{k} \rightarrow N$ that can be represented by a term of $T_{0}$ correspond exactly to primitive recursive functions. Not surprisingly, the term given in the above definition does not belong to $T_{0}$ but to $T_{1}$ (the outermost rec in the above definition has type int $\rightarrow$ int and thus has degree 1$)$.

\section{The higher-order Loop language}

In this section, we present the Loop ${ }^{\omega}$ language. First, we describe the syntax and the type system, then we detail the semantics of the language. Since the structured operational semantics is tailored for the lock-step simulation, we first present the natural semantics. Our purpose is to give evidence that the various syntactic constructs have the usual semantics. In particular, this semantics extends the usual semantics for first order LoOP programs with full-fledged higher-order procedures.

However natural semantics relates a program and an initial store directly to some final store and thus describes only the evaluation of terminating programs. In particular, it fails to distinguish between a non-terminating program and a run-time error. For this reason, we also define a transition semantics which refines the natural semantics. We use the transition semantics for proving the soundness of type systems and the simulation theorem (in order to derive that Loop ${ }^{\omega}$ programs are always terminating).

Both semantics are location-free semantics. The benefit is clear: this kind of semantics is simpler than a traditional two-level semantics (where the environment binds variables to locations and the store maps locations to values). The main drawback is the difficulty to account for advanced features such as variable aliasing. However, for our purpose, a location-free semantics is sufficient. A discussion on this subject can be found in [Plotkin, 1981] p. 70 (see also [Felleisen and Friedman, 1987], [Donahue, 1977] and [Reynolds, 1981]). 


\subsection{Syntax}

The syntax of imperative types and phrase types is the following:

$$
\begin{aligned}
\sigma, \tau & ::=\text { int } \mid \text { proc }(\text { in } \vec{\tau} ; \text { out } \vec{\sigma}) \\
\mathcal{T} & ::=\tau \mid \text { comm } \mid \text { seq }
\end{aligned}
$$

Note that we consider only two formal parameter modes in and out (borrowed from Ada [DOD, 1980]) which specify "abstractly" the direction of data flow between caller and callee (without implying a specific parameter-passing mechanism).

The raw syntax of imperative programs is given below. There is nothing particular to this syntax except that we annotate each block $\{s\}_{\vec{x}}$ with a list of variables $\vec{x}$ (see Remark 3.2). In the following grammar, $x, y, z$ range over a set of identifiers, $\bar{q}$ ranges over natural numbers (i.e., constant literals) and $\varepsilon$ denotes the empty sequence.

$$
\begin{aligned}
& \text { (command) } \quad c \quad::=\{s\}_{\vec{x}} \\
& \text { for } y:=1 \text { to } e\{s\}_{\vec{x}} \\
& |y:=e| \operatorname{inc}(y) \quad \mid \operatorname{dec}(y) \\
& p(\vec{e} ; \vec{y}) \\
& \text { (sequence) } \quad s::=\varepsilon \\
& \text { | } c ; s \\
& \text { | } \operatorname{cst} y=e ; s \\
& \text { | } \operatorname{var} y: \tau:=e ; s \\
& \text { (anonymous procedure) } \quad a::=\operatorname{proc}(\text { in } \vec{y}: \vec{\tau} ; \text { out } \vec{z}: \vec{\sigma})\{s\}_{\vec{z}} \\
& \text { (expression) } \quad e::=y \quad|\quad \bar{q}| \begin{array}{l}
a \\
\text { (procel }
\end{array} \\
& \text { (procedure) } \quad p::=y \quad \mid \quad a \\
& \text { (value) } \quad w::=\bar{q} \quad \mid a
\end{aligned}
$$

Remark 3.1. Note that the body of a procedure is annotated exactly by its out parameters. Besides, since anonymous procedures are not very popular in imperative languages, we use in the examples the more conventional notation for declaring local (named) procedures:

$$
\operatorname{proc} p(\text { in } \vec{y}: \vec{\tau} ; \text { out } \vec{z}: \vec{\sigma})\left\{s_{1}\right\}_{\vec{z}} ; s_{2}
$$

Following Landin's correspondence principle [Landin, 1964], this notation is defined as an abbreviation for a constant declaration:

$$
\text { cst } p=\operatorname{proc}(\text { in } \vec{y}: \vec{\tau} ; \text { out } \vec{z}: \vec{\sigma})\left\{s_{1}\right\}_{\vec{z}} ; s_{2}
$$

\subsection{Type system}

The type system of Loop ${ }^{\omega}$ may be seen as a simple effect system [Gifford and Lucassen, 1986, Talpin and Jouvelot, 1994] since it is able to guarantee the absence of side-effects, aliasing and fix-points in well-typed programs. Its main feature is the distinction between mutable variables and read-only variables. More formally, a typing environment has the form $\Gamma ; \Omega$ where $\Gamma$ and $\Omega$ are (possibly empty) lists of pairs $x$ : $\tau$ ( $x$ ranges over variables and $\tau$ over types). $\Gamma$ stands for read-only variables (constants and in parameters) and $\Omega$ stands for mutable variables (local variables and out parameters). The type system is given in Figure 3.1. As usual, we consider programs up to 


$$
\begin{aligned}
& \frac{x: \tau \in \Gamma ; \Omega}{\Gamma ; \Omega \vdash x: \tau} \\
& \text { (T.ENV) } \\
& \Gamma ; \Omega \vdash \bar{q}: \text { int } \\
& \text { (T.NUM) } \\
& \overline{\Gamma ; \Omega \vdash \varepsilon: \mathbf{s e q}} \\
& \text { (T.SEQ-I) } \\
& \frac{\Gamma ; \Omega \vdash c: \text { comm } \quad \Gamma ; \Omega \vdash s: \mathbf{s e q}}{\Gamma ; \Omega \vdash c ; s: \text { seq }} \\
& \frac{\Gamma ; \Omega \vdash e: \tau \quad \Gamma, y: \tau ; \Omega \vdash s: \mathbf{s e q}}{\Gamma ; \Omega \vdash \mathbf{c s t} y=e ; s: \mathbf{s e q}} \\
& \frac{\Gamma ; \Omega \vdash e: \tau \quad \Gamma ; y: \tau, \Omega \vdash s: \text { seq } \quad s \neq \varepsilon}{\Gamma ; \Omega \vdash \operatorname{var} y: \tau:=e ; s: \text { seq }} \\
& \frac{\vec{x} \subset \Omega \quad \Gamma ; \vec{x}: \vec{\sigma} \vdash s: \text { seq }}{\Gamma ; \Omega \vdash\{s\}_{\vec{x}}: \text { comm }} \\
& y: \text { int } \in \Omega \\
& \overline{\Gamma ; \Omega \vdash \operatorname{inc}(y): \mathbf{c o m m}} \\
& y: \text { int } \in \Omega \\
& \overline{\Gamma ; \Omega \vdash \operatorname{dec}(y): \text { comm }} \\
& \begin{array}{c}
y: \tau \in \Omega \quad \Gamma ; \Omega \vdash e: \tau \\
\Gamma ; \Omega \vdash y:=e: \text { comm }
\end{array} \\
& \frac{\vec{x} \subset \Omega \quad \Gamma ; \Omega \vdash e: \text { int } \quad \Gamma, y: \text { int } ; \vec{x}: \vec{\sigma} \vdash s: \mathbf{s e q}}{\Gamma ; \Omega \vdash \text { for } y:=1 \text { to } e\{s\}_{\vec{x}}: \mathbf{c o m m}} \\
& \vec{z} \neq \emptyset \quad \Gamma, \vec{y}: \vec{\tau} ; \vec{z}: \vec{\sigma} \vdash s: \text { seq } \\
& \Gamma ; \Omega \vdash \operatorname{proc}(\text { in } \vec{y}: \vec{\tau} ; \text { out } \vec{z}: \vec{\sigma})\{s\}_{\vec{z}}: \operatorname{proc}(\text { in } \vec{\tau} ; \text { out } \vec{\sigma}) \\
& \frac{\Gamma ; \Omega \vdash p \text { : proc }(\text { in } \vec{\tau} ; \text { out } \vec{\sigma}) \quad \Gamma ; \Omega \vdash \vec{e}: \vec{\tau} \quad \vec{r}: \vec{\sigma} \in \Omega}{\Gamma ; \Omega \vdash p(\vec{e} ; \vec{r}): \text { comm }} \quad \text { (T.CALL) }
\end{aligned}
$$

Figure 3.1. Imperative type system

renaming of bound variables, where the notion of free variable of a command is defined in the standard way.

Remark 3.2. (scoping rules). As usual for $C$-like languages, the scope of a constant (rule T.CST) or a variable (rule T.VAR) extends from the point of declaration to the end of the block containing the declaration. Moreover, the type system guarantees that in a block $\{s\}_{\vec{x}}$, the variables $\vec{x}$ are visible and they contain all the free mutable variables occurring in the sequence. For simplicity, we assume that these annotations are supplied by the programmer, but missing annotations can automatically be inferred as follows:

- a block or a loop is annotated by its free mutable variables,

- the body of a procedure is annotated by its out parameters.

Remark 3.3. (no aliasing). In order to avoid parameter-induced aliasing problems, we assume that all $r_{i}$ are pairwise distinct in rule (T.CALL). Indeed, we will see that in our semantics, mutable variables directly denote values and consequently different variables cannot refer to the same location in the store. However, aliasing is known to be problematic for most aspects of programming languages (see for instance [Gellerich and Plödereder, 2001] and [Filliâtre, 2003]) and we regard the absence of parameter-induced aliasing in $\operatorname{LoOP}^{\omega}$ more as a feature than as a limitation. 
Remark 3.4. (no side-effects). Rule (T.PROC) implies that the only mutable variables which may occur inside the body of a procedure are its out parameters and its local mutable variables. This is enough to guarantee the absence of side-effects (i.e., modification of non-local variables). The purpose of this restriction is mainly to simplify the semantics of the language. However, side-effects can still be simulated in most cases by passing the non-local variable as an explicit in out parameter. This simulation is often referred to as the "state-passing transform" (see for instance [Filinski, 1994] and [Wadler, 1990]).

Remark 3.5. (no fix-points). Rule (T.PROC) also forbids the reading of non-local mutable variables: this is necessary to prevents the definition of fix-points in the language. Indeed, there is a well-known technique called "tying the recursive knot" [Landin, 1964] which takes advantage of higher-order mutable variables (or function pointers) to define arbitrary recursive functions. This technique is used for instance in Scheme's semantics of the letrec construct [Kelsey et al., 1998]. Here is such a definition of a fix-point in the LoOP $^{\omega}$ syntax, where $f i x$ : proc (out int) is a mutable variable, but this command is not typable in $\operatorname{LoOP}^{\omega}$ (since $f x$ occurs in the body of $f$ ):

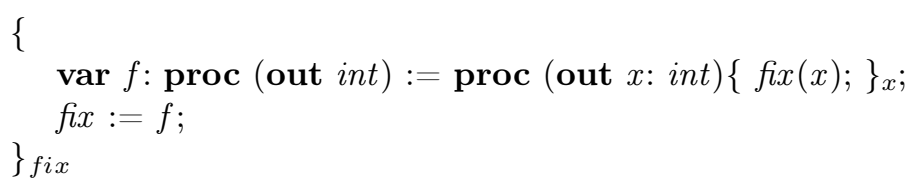

On the other hand, local procedures are not required to be closed: non-local read-only variable (such as in parameters of enclosing procedures) are allowed. We shall see that this is sufficient to encode a pure functional language.

\subsection{Natural semantics}

Since the evaluation relation in only defined for well-typed states, we also need to define the notion of well-typed store.

Definition 3.6. A store $\mu$ is a finite mapping from (mutable) variables to closed imperative values (i.e., integer literals and procedures). A state is a pair $(c, \mu)$ consisting of a command $c$ and $\mu$.

Definition 3.7. (store typing). We say that $\mu$ is typable in $\Omega$, which we write as $\Omega \vdash \mu$, if and only if for all $x: \tau \in \Omega, x \in \operatorname{dom}(\mu)$ and $\emptyset ; \emptyset \vdash \mu(x): \tau$.

Definition 3.8. (state typing). We say that a state $(c, \mu)$ is typable in $\Omega$, which we write as $\Omega \vdash(c, \mu)$, if and only if $\emptyset ; \Omega \vdash c$ : comm and $\Omega \vdash \mu$.

Note that expressions do not require any evaluation (since they are either variables or values), but only fetching the corresponding value from the store whenever the expression is a mutable variable. We introduce thus the following notation:

Notation 3.9. Given a store $\mu$, let $\varphi_{\mu}$ be the trivial extension of $\mu$ to expressions defined as follows $\varphi_{\mu}(x)=\mu(x)$ if $x$ is a variable and $\varphi_{\mu}(w)=w$ otherwise. In the sequel, we write $e={ }_{\mu} w$ for $\varphi_{\mu}(e)=w$.

In order to assign default values to out parameters, we define a closed imperative value for each imperative type.

Definition 3.10. For each type $\sigma$, we define inductively a default closed imperative value $\epsilon(\sigma)$ as follows:

$$
\begin{aligned}
& -\epsilon(\text { int })=0 \\
& \text { - } \epsilon(\text { proc }(\text { in } \vec{\tau} ; \text { out } \vec{\sigma}))=\text { proc }(\text { in } \vec{y}: \vec{\tau} \text {; out } z: \vec{\sigma})\{\}_{\vec{z}}
\end{aligned}
$$


Lemma 3.11. The typing judgment $\emptyset ; \emptyset \vdash \epsilon(\sigma): \sigma$ is derivable.

Remark 3.12. Default values could be dispensed with using standard data-flow analysis (see [Appel, 1998] for instance). This technique (also called liveness analysis) allows to determine whether there is a potential execution path on which a variable is used before it has been assigned an initial value. We preferred not to complicate the static analysis of LoOP $^{\omega}$ programs although such an analysis is certainly convenient from a practical standpoint.

Remark 3.13. In the sequel, we shall allow for uninitialized local variables: they are assumed to be implicitly initialized by the default value corresponding to their type. More precisely, $\operatorname{var} y: \tau ; s$ is an abbreviation for: var $y: \tau:=\epsilon(\tau) ; s$.

Notation 3.14. Let $c$ be a command. We write $c[x \leftarrow w]$ for the substitution of a readonly variable $x$ by a closed imperative value $w$ and $c[y \hookleftarrow z]$ for the renaming of a mutable variable $y$ by a mutable variable $z$. The formal definitions are given in Appendix B.

We are now ready to define the natural semantics of Loop ${ }^{\omega}$. The inductive definition of the evaluation relation written $\Downarrow$ is summarized in Figure 3.2 (where $\mu[y \leftarrow w]$ denotes the store obtained by replacing the value of variable $y$ in $\mu$ by $w$ and $(\mu, y \leftarrow w)$ denotes the store obtained by extending the store $\mu$ with a new variable $y$ mapped to $w$ ).

$$
\begin{aligned}
& \frac{(s, \mu) \Downarrow \mu^{\prime}}{\left(\{s\}_{\vec{z}}, \mu\right) \Downarrow \mu^{\prime}} \\
& \text { (N.BLOCK) } \\
& \frac{(s, \mu) \Downarrow \mu^{\prime}}{\left(\left(\{\}_{\vec{z}} ; s\right), \mu\right) \Downarrow \mu^{\prime}} \\
& \text { (N.SEQ-I) } \\
& \frac{(c, \mu) \Downarrow \mu^{\prime} \quad\left(s, \mu^{\prime}\right) \Downarrow \mu^{\prime \prime}}{((c ; s), \mu) \Downarrow \mu^{\prime \prime}} \\
& \frac{(s, \mu) \Downarrow \mu^{\prime}}{\left(\left(\{\operatorname{var} y: \tau:=e ;\}_{\vec{z}} ; s\right), \mu\right) \Downarrow \mu^{\prime}} \\
& \frac{\left.e={ }_{\mu} w \quad(s,(\mu, y \leftarrow w)) \Downarrow\left(\mu^{\prime}, y \leftarrow w^{\prime}\right)\right)}{((\operatorname{var} y: \tau:=e ; s), \mu)) \Downarrow \mu^{\prime}} \\
& \frac{e={ }_{\mu} w \quad(s, \mu[y \leftarrow w]) \Downarrow \mu^{\prime}}{((y:=e ; s), \mu) \Downarrow \mu^{\prime}} \\
& \frac{\mu(y)=\bar{q}}{(\operatorname{inc}(y), \mu)) \Downarrow \mu[y \leftarrow \overline{q+1}]} \\
& \frac{\mu(y)=\bar{q}}{(\operatorname{dec}(y), \mu) \Downarrow \mu[y \leftarrow \overline{q-1}]} \\
& \frac{\vec{e}={ }_{\mu} \vec{w}, p={ }_{\mu} \operatorname{proc}(\text { in } \vec{y}: \vec{\sigma} ; \text { out } \vec{z}: \vec{\tau})\{s\}_{\vec{z}} \quad\left(\{s[\vec{y} \leftarrow \vec{w}][\vec{z} \hookleftarrow \vec{r}]\}_{\vec{r}}, \mu[\vec{r} \leftarrow \epsilon(\vec{\tau})]\right) \Downarrow \mu^{\prime}}{(p(\vec{e} ; \vec{r}), \mu) \Downarrow \mu^{\prime}} \\
& \frac{e={ }_{\mu} w \quad(s[y \leftarrow w], \mu) \Downarrow \mu^{\prime}}{((\mathbf{c s t} y=e ; s), \mu) \Downarrow \mu^{\prime}} \\
& \frac{e={ }_{\mu} \overline{0}}{\left(\text { for } y:=1 \text { to } e\{s\}_{\vec{z}}, \mu\right) \Downarrow \mu} \\
& \frac{e={ }_{\mu} \overline{q+1} \quad\left(\text { for } y:=1 \text { to } \bar{q}\{s\}_{\vec{z}}, \mu\right) \Downarrow \mu^{\prime} \quad\left(\{s\}_{\vec{z}}[y \leftarrow \overline{q+1}], \mu^{\prime}\right) \Downarrow \mu^{\prime \prime}}{\left(\text { for } y:=1 \text { to } e\{s\}_{\vec{z}}, \mu\right) \Downarrow \mu^{\prime \prime}}
\end{aligned}
$$

Figure 3.2. Natural semantics 
Remark 3.15. We rely on the renaming and substitution meta-operations in rule (N.CALL) in order to avoid building closures in the semantics. Besides, in parameters are passed by copy (i.e., by value) whereas out parameters are passed by reference. Note that since parameter-induced aliasing is forbidden, this latter choice has no consequence on the denotational semantics of the language (but it is important from a complexity standpoint).

\subsection{Transition semantics}

As expected, the transition semantics of $\mathrm{LOOP}^{\omega}$ is given by a transition system which defines inductively a binary relation between states [Plotkin, 1981]. The main design choices were influenced by the expected cost of each command. For instance, this explains the rule (S.ASSIGN) where the assignment is dealt with directly inside the sequence. Similarly, the notation $={ }_{\mu}$ allows us to hide the cost of fetching the value of a variable from the store. The transition semantics is summarized in Figure 3.3.

Remark 3.16. This semantics is clearly deterministic since there is always at most one rule which can be applied (depending on the content of the store and the shape of the command). Moreover, the only case where no rule can be applied corresponds to the final state (when the program is reduced to an empty block).

Remark 3.17. It is worth mentioning that rules (S.VAR-I) and (S.VAR-II) allows to give a simple semantics to local variable without dealing with an explicit stack. Although this is usual in natural semantics, this technique is not widespread in transition semantics. The ingenious idea consisting in updating a local variable directly in the source (in rule S.VARII) is attributed to Eugene Fink in [Reynolds, 1998] p. 130. This technique is however well-known in functional language semantics [Felleisen and Friedman, 1987].

As expected, the "subject reduction" property holds for the transition semantics. The proof of the following theorem is given in Appendix B.

Theorem 3.18. For any environment $\Omega$ and any state $(c, \mu)$, we have that $\emptyset ; \Omega \vdash(c, \mu)$ and $(c, \mu) \mapsto\left(c^{\prime}, \mu^{\prime}\right)$ implies $\emptyset ; \Omega \vdash\left(c^{\prime}, \mu^{\prime}\right)$ and $\operatorname{dom}(\mu)=\operatorname{dom}\left(\mu^{\prime}\right)$.

Moreover, the equivalence of the natural and transition semantics for Loop ${ }^{\omega}$ can be proved by establishing the following two usual lemmas.

Lemma 3.19. The relation $\left(\{s\}_{\vec{x}}, \mu\right) \mapsto^{\star}\left(\{\}_{\vec{x}}, \mu^{\prime}\right)$ is closed under the defining conditions of the $\Downarrow$ relation.

Lemma 3.20. The $\Downarrow$ relation is closed under head expansion: if $\left(\{s\}_{\vec{x}}, \mu\right) \mapsto\left(\left\{s^{\prime}\right\}_{\vec{x}}, \mu^{\prime}\right)$ and $\left(\left\{s^{\prime}\right\}_{\vec{x}}, \mu^{\prime}\right) \Downarrow \mu^{\prime \prime}$ then $\left(\{s\}_{\vec{x}}, \mu\right) \Downarrow \mu^{\prime \prime}$.

\section{Lock-step simulation}

In this section, we show how to translate a $\operatorname{LOOP}^{\omega}$ program into a term of System $\mathrm{T}$ and we prove the simulation theorem. Then we exhibit a hierarchy of fragments Loop ${ }^{n}$ which is an imperative counterpart of the fragments $\mathrm{T}_{n}$ of Gödel System T. Finally, we introduce the notion of "singular" LoOP ${ }^{\omega}$ program whose translation does not require the product type in System T.

\subsection{Translation}

In order to translate default imperative values, we shall need corresponding default functional values. 


$$
\begin{aligned}
& \frac{(s, \mu) \mapsto\left(s^{\prime}, \mu^{\prime}\right)}{\left(\{s\}_{\vec{z}}, \mu\right) \mapsto\left(\left\{s^{\prime}\right\}_{z}, \mu^{\prime}\right)} \\
& \left(\left(\{\}_{\vec{z}} ; s\right), \mu\right) \mapsto(s, \mu) \quad \text { (S.SEQ-I) } \\
& \frac{(c, \mu) \mapsto\left(c^{\prime}, \mu^{\prime}\right)}{((c ; s), \mu) \mapsto\left(\left(c^{\prime} ; s\right), \mu^{\prime}\right)} \quad \text { (S.SEQ-II) } \\
& \frac{e={ }_{\mu} w \quad(s,(\mu, y \leftarrow w)) \mapsto\left(\varepsilon,\left(\mu^{\prime}, y \leftarrow w^{\prime}\right)\right)}{((\operatorname{var} y: \tau:=e ; s), \mu) \mapsto\left(\varepsilon, \mu^{\prime}\right)} \quad \text { (S.VAR-I) } \\
& \frac{e={ }_{\mu} w \quad(s,(\mu, y \leftarrow w)) \mapsto\left(s^{\prime},\left(\mu^{\prime}, y \leftarrow w^{\prime}\right)\right)}{((\operatorname{var} y: \tau:=e ; s), \mu) \mapsto\left(\left(\operatorname{var} y: \tau:=w^{\prime} ; s^{\prime}\right), \mu^{\prime}\right)} \\
& \frac{e={ }_{\mu} w}{((y:=e ; s), \mu) \mapsto(s, \mu[y \leftarrow w])} \quad \text { (S.ASSIGN) } \\
& \frac{\mu(y)=\bar{q}}{(\operatorname{inc}(y), \mu) \mapsto(y:=\overline{q+1}, \mu)} \quad \text { (S.INC) } \\
& \frac{\mu(y)=\bar{q}}{(\operatorname{dec}(y), \mu) \mapsto(y:=\overline{q-1}, \mu)} \\
& \begin{array}{ll}
\vec{e}={ }_{\mu} \vec{w} & p={ }_{\mu} \text { proc }(\text { in } \vec{y}: \vec{\sigma} ; \text { out } \vec{z}: \vec{\tau})\{s\}_{\vec{z}}
\end{array} \quad \text { (S.CALL) } \\
& \frac{e={ }_{\mu} w}{((\mathbf{c s t} y=e ; s), \mu) \mapsto(s[y \leftarrow w], \mu)} \quad \text { (S.CST) } \\
& \frac{e={ }_{\mu} \overline{0}}{\left(\text { for } y:=1 \text { to } e\{s\}_{\vec{z}}, \mu\right) \mapsto\left(\{\}_{\vec{z}}, \mu\right)} \quad \text { (S.FOR-I) } \\
& \frac{e={ }_{\mu} \overline{q+1}}{\left(\text { for } y:=1 \text { to } e\{s\}_{\vec{z}}, \mu\right) \mapsto\left(\left\{\text { for } y:=1 \text { to } \bar{q}\{s\}_{\vec{z}} ; s[y \leftarrow \overline{q+1}]\right\}_{\vec{z}}, \mu\right)} \quad \text { (S.FOR-II) }
\end{aligned}
$$

Figure 3.3. Transition semantics

Definition 4.1. For each type $\sigma$, we define inductively a default closed value $\delta(\sigma)$ as follows:

$$
\begin{array}{ll}
- & \delta(i n t)=0 \\
- & \delta(\sigma \rightarrow \tau)=\mathbf{f n}(x: \sigma) \Rightarrow \delta(\tau) \\
- & \delta\left(\sigma_{1} \times \ldots \times \sigma_{n}\right)=\left(\delta\left(\sigma_{1}\right), \ldots, \delta\left(\sigma_{n}\right)\right)
\end{array}
$$

We also write $\delta(\vec{\sigma})$ as an abbreviation for $\left(\delta\left(\sigma_{1}\right), \ldots, \delta\left(\sigma_{n}\right)\right)$.

Lemma 4.2. The typing judgment $\vdash \delta(\sigma): \sigma$ is derivable.

We are now ready to define the translation of imperative types. Note that the translation of a procedure type encode exactly the data-flow specified by the formal parameter modes in and out (and this is sufficient since side-effects are not allowed).

Definition 4.3. The translations $\sigma^{\star}$ of an imperative type $\sigma$ is defined inductively as follows:

$$
\begin{aligned}
& -\quad \text { int } t^{\star}=\text { int } \\
& -\quad \operatorname{proc}(\text { in } \vec{\sigma} ; \text { out } \vec{\tau})^{\star}=\left(\vec{\sigma}^{\star}\right)^{\star} \rightarrow\left(\vec{\tau}^{\star}\right)^{\times}
\end{aligned}
$$

Moreover, if $\Gamma$ is $x_{1}: \tau_{1}, \ldots, x_{n}: \tau_{n}$, we define $\Gamma^{\star}$ as $x_{1}: \tau_{1}^{\star}, \ldots, x_{n}: \tau_{n}^{\star}$.

The intuition behind the translation of imperative programs is the following: a block $\left\{c_{1} ; \ldots ; c_{n}\right\}_{\vec{x}}$ is translated into:

$$
\text { let } \vec{x}_{1}=c_{1}^{\star} \text { in ... let } \vec{x}_{n}=c_{n}^{\star} \text { in } \vec{x}
$$


where each $\vec{x}_{i} \subseteq \vec{x}$ corresponds to the "output" of command $c_{i}$ and $\vec{x}$ is the output of the block. Note in particular that the same identifier is used again and again in order to simulate imperative updates. For instance, the block $\{\operatorname{inc}(x) ; \operatorname{inc}(x)\}_{x}$ is translated as:

$$
\text { let } x=\operatorname{succ}(x) \text { in let } x=\operatorname{succ}(x) \text { in } x
$$

Let us now give the formal definition of the translation and then present a complete example.

Definition 4.4. For any expression e, block $b$, sequence $s$ and variables $\vec{x}$, the translations $e^{\star}, b^{\star}$ and $(s)_{\vec{x}}^{\star}$ into terms of System $T$ are defined by mutual induction as follows:

$$
\begin{array}{ll}
- & \bar{n}^{\star}=S^{n}(0) \\
- & y^{\star}=y \\
- & \left(\operatorname{proc}(\text { in } \vec{y}: \vec{\sigma} ; \text { out } \vec{z}: \vec{\tau})\{s\}_{\vec{z}}\right)^{\star}=\mathbf{f n}\left(\vec{y}: \vec{\sigma}^{\star}\right) \Rightarrow\{s\}_{\vec{z}}^{\star}\left[\delta\left(\vec{\tau}^{\star}\right) / \vec{z}\right] \\
- & \{s\}_{\vec{x}}^{\star}=(s)_{\vec{x}}^{\star} \\
- & (\varepsilon)_{\vec{x}}^{\star}=\vec{x} \\
- & (\operatorname{var} y: \tau:=e ; s)_{\vec{x}}^{\star}=(s)_{\vec{x}}^{\star}\left[e^{\star} / y\right] \\
- & (\operatorname{cst} y=e ; s)_{\vec{x}}^{\star}=\operatorname{let} y=e^{\star} \operatorname{in}(s)_{\vec{x}}^{\star} \\
- & (y:=e ; s)_{\vec{x}}^{\star}=\operatorname{let} y=e^{\star} \operatorname{in}(s)_{\vec{x}}^{\star} \\
- & (\operatorname{inc}(y) ; s)_{\vec{x}}^{\star}=\operatorname{let} y=\operatorname{succ}(y) \text { in }(s)_{\vec{x}}^{\star} \\
- & (\operatorname{dec}(y) ; s)_{\vec{x}}^{\star}=\operatorname{let} y=\operatorname{pred}(y) \text { in }(s)_{\vec{x}}^{\star} \\
- & (p(\vec{e} ; \vec{z}) ; s)_{\vec{x}}^{\star}=\operatorname{let} \vec{z}=p^{\star} \vec{e}^{\star} \operatorname{in}(s)_{\vec{x}}^{\star} \\
- & \left(\left\{s_{1}\right\}_{\vec{z}} ; s_{2}\right)_{\vec{x}}^{\star}=\operatorname{let} \vec{z}=\left\{s_{1}\right\}_{\vec{z}}^{\star} \operatorname{in}\left(s_{2}\right)_{\vec{x}}^{\star} \\
- & \left(\text { for } y:=1 \text { to } e\left\{s_{1}\right\}_{\vec{z}} ; s_{2}\right)_{\vec{x}}^{\star}=\operatorname{let} \vec{z}=\operatorname{rec}\left(e^{\star}, \vec{z}, \lambda y \cdot \lambda \vec{z} \cdot\left\{s_{1}\right\}_{\vec{z}}^{\star}\right) \text { in }\left(s_{2}\right)_{\vec{x}}^{\star}
\end{array}
$$

Remark 4.5. It is possible to factor out the translation of commands in the above definition. Indeed, the translation of a non-empty block $\{c ; s\}_{\vec{x}}$ always follows the same pattern: $\{c ; s\}_{\vec{x}}^{\star}=$ let $\vec{z}=c^{\star}$ in $\{s\}_{\vec{x}}^{\star}$ where $\vec{z}$ is a list of "output" variables of depending on the command $c$. The translation $c^{\star}$ of a command $c$ together with its output variables $\mathcal{O}(c)$ are summarized in the following table:

\begin{tabular}{|l|l|l|}
\hline$c$ & $c^{\star}$ & $\mathcal{O}(c)$ \\
\hline$y:=e$ & $e^{\star}$ & $y$ \\
\hline $\operatorname{inc}(y)$ & $\operatorname{succ}(y)$ & $y$ \\
\hline $\operatorname{dec}(y)$ & $\operatorname{pred}(y)$ & $y$ \\
\hline$p(\vec{e} ; \vec{z})$ & $p^{\star} \vec{e}^{\star}$ & $\vec{z}$ \\
\hline$\{s\}_{\vec{z}}$ & $\{s\}_{\vec{z}}^{\star}$ & $\vec{z}$ \\
\hline for $y:=1$ to $e\{s\}_{\vec{z}}$ & $\operatorname{rec}\left(e^{\star}, \vec{z}, \lambda y \cdot \lambda \vec{z} \cdot\{s\}_{\vec{z}}^{\star}\right)$ & $\vec{z}$ \\
\hline
\end{tabular}

Lemma 4.6. For any imperative type $\sigma$, we have $\epsilon(\sigma)^{\star}=\delta\left(\sigma^{\star}\right)$.

The following theorem states that translation ()$^{\star}$ is type-preserving (its proof is given in Appendix C).

Theorem 4.7. For any environments $\Gamma$ and $\Omega$, any expression e and any block $\{s\}_{\vec{x}}$ we have:

- $\quad \Gamma ; \Omega \vdash e: \tau$ implies $\Gamma^{\star}, \Omega^{\star} \vdash e^{\star}: \tau^{\star}$

- $\quad \Gamma ; \Omega, \vec{x}: \vec{\sigma} \vdash s$ : seq implies $\Gamma^{\star}, \Omega^{\star}, \vec{x}: \vec{\sigma}^{\star} \vdash(s)_{\vec{x}}^{\star}:\left(\vec{\sigma}^{\star}\right)^{\star}$

- $\quad \Gamma ; \vec{x}: \vec{\sigma} \vdash\{s\}_{\vec{x}}:$ comm implies $\Gamma^{\star}, \vec{x}: \vec{\sigma}^{\star} \vdash\{s\}_{\vec{x}}^{\star}:\left(\vec{\sigma}^{\star}\right)^{\star}$ 


\subsection{Example: the Ackermann function}

Here is an implementation of the Ackermann function as an anonymous procedure of Loop $^{\omega}$. This program was actually programmed by hand, but we shall see in Section 5 how to get almost the same program from the definition of ack in System T.

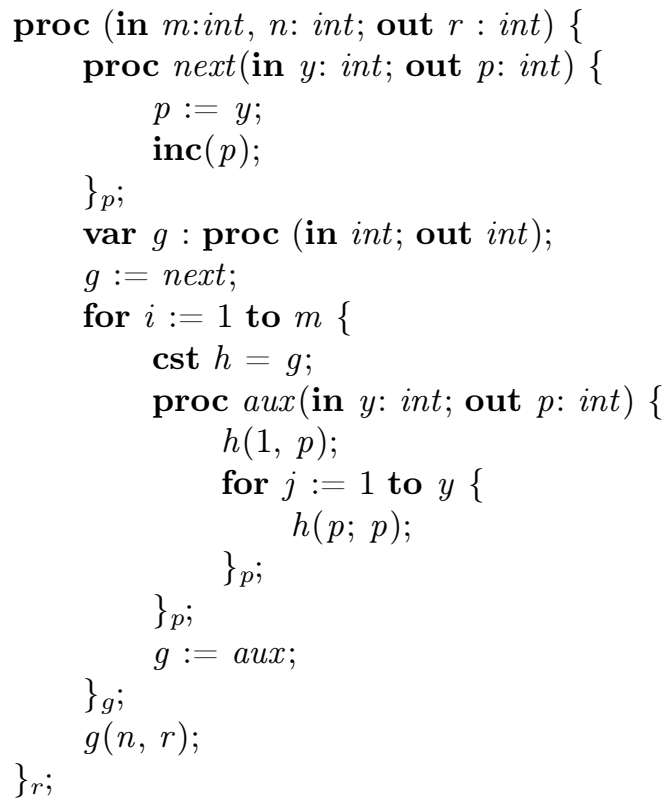

By applying translation ()$^{\star}$, we obtain the following anonymous function. For clarity, we use Standard ML derived forms [Milner et al., 1997] for declaring several functions (keyword fun) and values (keyword val) within a unique let.

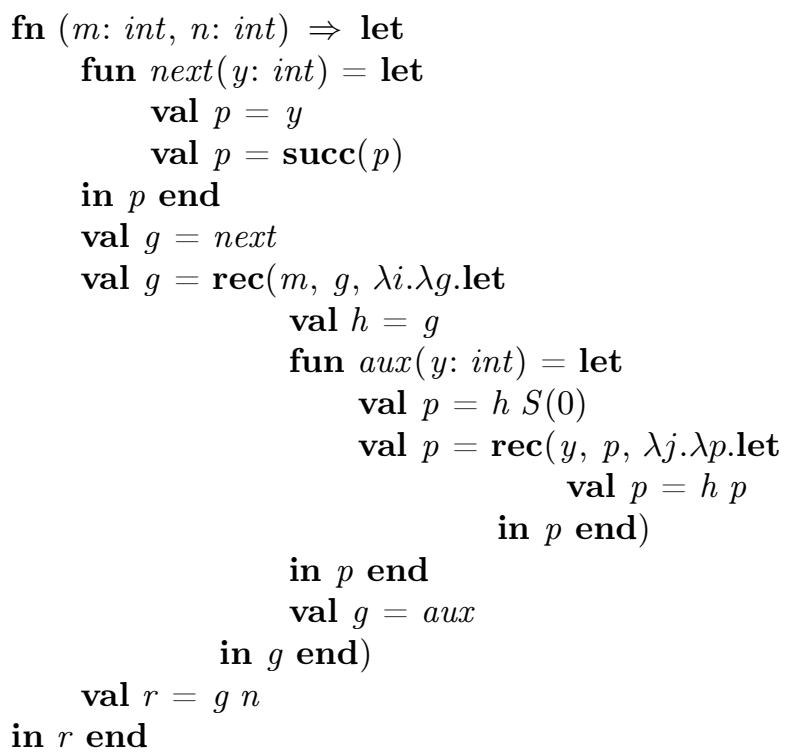

Note that although we could prove by hand that this functional program is equivalent to the term ack given in Section 2.1, we shall see that this property is mostly an application of the main theorem of Section 5 .

\subsection{Simulation theorem}

Let us prove the main theorem which states that for any block $\{s\}_{\vec{x}}$, the evaluation of $\{s\}_{\vec{x}}$ runs in lock-step with the reduction of $\{s\}_{\vec{x}}^{\star}$. We first need some preliminary substitution lemmas: 
Notation 4.8. If $\mu$ is a store and $\vec{x} \subset \operatorname{dom}(\mu)$, we write $\mu(\vec{x})$ for $\left(\mu\left(x_{1}\right), \ldots, \mu\left(x_{n}\right)\right)$. Moreover, if $\vec{v}=\left(v_{1}, \ldots, v_{k}\right)$ we write $\vec{v}^{\star}$ for the tuple $\left(v_{1}^{\star}, \ldots, v_{k}^{\star}\right)$.

Lemma 4.9. For any block $\{s\}_{\vec{z}}$ such that $\Gamma ; \vec{z}: \vec{\tau} \vdash\{s\}_{\vec{z}}$ : comm, the following equality holds: $\{s\}_{\vec{z}}^{\star}[\delta(\vec{\tau}) / \vec{z}]=\{s[\vec{z} \hookleftarrow \vec{r}]\}_{\vec{r}}^{\star}[\delta(\vec{\tau}) / \vec{r}]$.

Lemma 4.10. For any command $c$, any read-only variable $x$ and any closed imperative value $w$, we have $(c[\vec{x} \leftarrow \vec{w}])^{\star}=c^{\star}\left[\vec{w}^{\star} / \vec{x}\right]$.

Lemma 4.11. If $e={ }_{\mu} w$ then either $e=w$ or $e$ is some variable $x_{i}$ with $\mu\left(x_{i}\right)=w$ and then $c^{\star}\left[\mu(\vec{x})^{\star} / \vec{x}\right]=c^{\star}\left[w^{\star} / x_{i}\right]\left[\mu(\vec{x})^{\star} / \vec{x}\right]$.

Theorem 4.12. For any well-typed state $(s, \mu)$, if $\vec{x}=\operatorname{dom}(\mu)$ and $\vec{z} \subseteq \vec{x}$ we have:

$$
(s, \mu) \mapsto\left(s^{\prime}, \mu^{\prime}\right) \text { implies }(s)_{\vec{z}}^{\star}\left[\mu(\vec{x})^{\star} / \vec{x}\right] \rightsquigarrow\left(s^{\prime}\right)_{z}^{\star}\left[\mu^{\prime}(\vec{x})^{\star} / \vec{x}\right]
$$

Proof. By induction on the derivation of $(s, \mu) \mapsto\left(s^{\prime}, \mu^{\prime}\right)$. For brevity, we write $\vec{v}$ for $\mu(\vec{x})$ and $\vec{v}^{\prime}$ for $\mu^{\prime}(\vec{x})$.

- $(\mathrm{S} . \mathrm{BLOCK})$

Indeed:

$$
\frac{(s)_{\vec{z}}^{\star}\left[\vec{v}^{\star} / \vec{x}\right] \rightsquigarrow\left(s^{\prime}\right)_{\bar{z}}^{\star}\left[\vec{v}^{\prime \star} / \vec{x}\right]}{\left.\{s\}_{\vec{z}}^{\star}\left[\vec{v}^{\star} / \vec{x}\right]\right) \rightsquigarrow\left\{s^{\prime}\right\}_{\vec{z}}^{\star}\left[\vec{v}^{\prime \star} / \vec{x}\right]}
$$

$$
\begin{aligned}
\{s\}_{\vec{z}}^{\star} & {\left[\vec{v}^{\star} / \vec{x}\right] } \\
& =(s)_{\frac{\star}{z}}^{\star}\left[\vec{v}^{\star} / \vec{x}\right] \\
& \left.\rightsquigarrow\left(s^{\prime}\right)_{\vec{z}}^{\star}\left[\vec{v}^{\prime \star} / \vec{x}\right] \text { by induction hypothesis (since } \vec{z} \subseteq \vec{x}\right) \\
& =\left\{s^{\prime}\right\}_{\vec{z}}^{\star}\left[\vec{v}^{\prime \star} / \vec{x}\right]
\end{aligned}
$$

- (S.SEQ-I)

Indeed:

$$
\left(\{\}_{\vec{y}} ; s\right)_{\vec{z}}^{\star}\left[\vec{v}^{\star} / \vec{x}\right] \rightsquigarrow(s)_{\vec{z}}^{\star}\left[\vec{v}^{\star} / \vec{x}\right]
$$

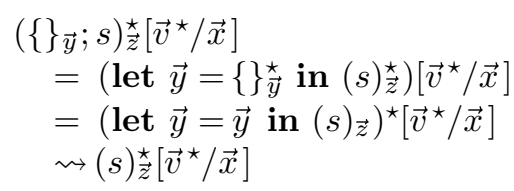

- $\quad$ (S.SEQ-II)

$$
\frac{c^{\star}\left[\vec{v}^{\star} / \vec{x}\right] \rightsquigarrow c^{\prime \star}\left[\vec{v}^{\prime \star} / \vec{x}\right]}{(c ; s)_{\vec{z}}^{\star}\left[\vec{v}^{\star} / \vec{x}\right] \rightsquigarrow\left(c^{\prime} ; s\right)_{\frac{\pi}{z}}\left[\vec{v}^{\prime \star} / \vec{x}\right]}
$$

Indeed, if $\vec{r}=\mathcal{O}(c)$ as defined in Remark 4.5, we have:

$$
\begin{aligned}
(c ; s) & \frac{\star}{\vec{z}}\left[\vec{v}^{\star} / \vec{x}\right] \\
& =\left(\text { let } \vec{r}=c^{\star} \text { in }(s)_{\vec{z}}^{\star}\right)\left[\vec{v}^{\star} / \vec{x}\right] \\
& =\left(\text { let } \vec{r}=c^{\star}\left[\vec{v}^{\star} / \vec{x}\right] \text { in }(s)_{\vec{z}}^{\star}\right)\left[\vec{v}^{\star} / \vec{x}\right] \\
& \rightsquigarrow\left(\text { let } \vec{r}=c^{\prime \star}\left[\vec{v}^{\prime \star} / \vec{x}\right] \text { in }(s)_{\vec{z}}^{\star}\right)\left[\vec{v}^{\star} / \vec{x}\right] \text { by induction hypothesis } \\
& =\left(\text { let } \vec{r}=c^{\prime \star}\left[\vec{v}^{\prime \star} / \vec{x}\right] \text { in }(s)_{\vec{z}}^{\star}\right)\left[\vec{v}^{\prime \star} / \vec{x}\right] \text { since } \mu^{\star}(y)=\mu^{\prime \star}(y) \text { for any } y \notin \vec{r} \\
& =\left(\text { let } \vec{r}=c^{\prime \star} \text { in }(s)_{\vec{z}}^{\star}\right)\left[\vec{v}^{\prime \star} / \vec{x}\right] \\
& =\left(c^{\prime} ; s\right)_{\vec{z}}^{\star}\left[\vec{v}^{\prime \star} / \vec{x}\right]
\end{aligned}
$$

- (S.VAR-I)

$$
\frac{(s)_{\vec{z}}^{\star}\left[v^{\star} / \vec{x}, w^{\star} / y\right] \rightsquigarrow(\varepsilon)_{\vec{z}}^{\star}\left[v^{\prime \star} / \vec{x}, w^{\prime \star} / y\right]}{(\operatorname{var} y: \tau:=e ; s)_{\vec{z}}^{\star}\left[\vec{v}^{\star} / \vec{x}\right] \rightsquigarrow(\varepsilon)_{\vec{z}}^{\star}\left[\vec{v}^{\prime \star} / \vec{x}\right]}
$$

Since $e={ }_{\mu} w$, by lemma 4.11: 


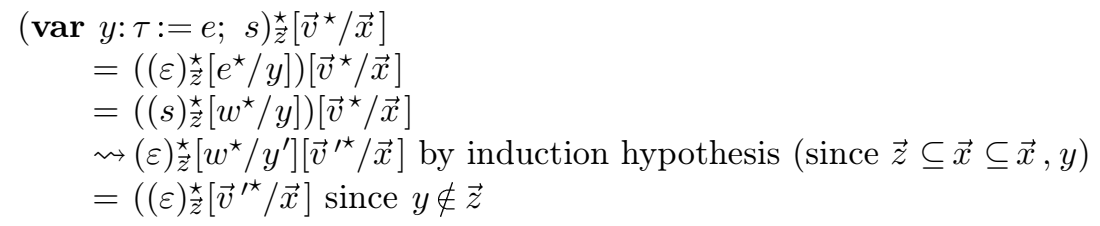

- $\quad(\mathrm{S} . \mathrm{VAR}-\mathrm{II})$

$$
\frac{(s)_{\vec{z}}^{\star}\left[v^{\star} / \vec{x}, w^{\star} / y\right] \rightsquigarrow\left(s^{\prime}\right)_{\vec{z}}^{\star}\left[v^{\prime \star} / \vec{x}, w^{\prime \star} / y\right]}{(\operatorname{var} y: \tau:=e ; s)_{\vec{z}}^{\star}\left[\vec{v}^{\star} / \vec{x}\right] \rightsquigarrow\left(\operatorname{var} y: \tau:=w^{\prime} ; s^{\prime}\right)_{\vec{z}}^{\star}\left[\vec{v}^{\prime \star} / \vec{x}\right]}
$$

Since $e={ }_{\mu} w$, by lemma 4.11:

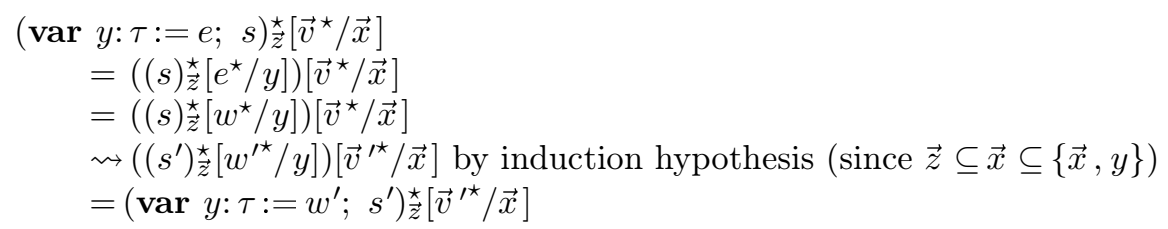

- (S.ASSIGN)

$$
(y:=e ; s)_{\vec{z}}^{\star}\left[\vec{v}^{\star} / \vec{x}\right] \rightsquigarrow(s)_{\vec{z}}^{\star}\left[\vec{v}^{\prime \star} / \vec{x}\right]
$$

with $\vec{v}^{\prime}=\mu[y \leftarrow w](\vec{x})$. Since $e={ }_{\mu} w$, by lemma 4.11:

$$
\begin{aligned}
(y:= & e ; s)_{\vec{z}}^{\star}\left[\vec{v}^{\star} / \vec{x}\right] \\
& =\left(\text { let } y=e^{\star} \text { in }(s)_{\vec{z}}^{\star}\right)\left[\vec{v}^{\star} / \vec{x}\right] \\
& =\left(\text { let } y=w^{\star} \text { in }(s)_{\vec{z}}^{\star}\right)\left[\vec{v}^{\star} / \vec{x}\right] \\
& \rightsquigarrow(s)_{\vec{z}}^{\star}\left[w^{\star} / y\right]\left[\vec{v}^{\star} / \vec{x}\right] \\
& =(s)_{\vec{z}}^{\star}\left[\vec{v}^{\prime \star} / \vec{x}\right]
\end{aligned}
$$

- $($ S.INC)

Indeed, since $\mu(y)=\bar{q}$ :

$$
(\operatorname{inc}(y))^{\star}\left[\vec{v}^{\star} / \vec{x}\right] \rightsquigarrow \overline{q+1}{ }^{\star}\left[\vec{v}^{\star} / \vec{x}\right]
$$

$$
\begin{aligned}
(\operatorname{inc} & (y))^{\star}\left[\vec{v}^{\star} / \vec{x}\right] \\
& =\operatorname{succ}(y)\left[\vec{v}^{\star} / \vec{x}\right] \\
& =\operatorname{succ}\left(S^{q}(0)\right) \\
& \left.\rightsquigarrow\left(S^{q+1}(0)\right)\right) \\
& =\frac{q+1}{q+1}
\end{aligned}
$$

- (S.DEC) This case is similar to (S.INC).

- (S.CALL)

$$
p(\vec{e}, \vec{r})^{\star}\left[\vec{v}^{\star} / \vec{x}\right] \rightsquigarrow\{s[\vec{z} \hookleftarrow \vec{r}][\vec{y} \leftarrow \vec{w}]\}_{\vec{r}}^{\star}\left[\vec{v}^{\prime \star} / \vec{x}\right]
$$

where $\vec{v}^{\prime}=\mu^{\prime}(\vec{x})=\mu[\vec{r} \leftarrow \epsilon(\vec{\tau})](\vec{x})$. By Lemma 4.11, since $\vec{e}={ }_{\mu} \vec{w}$ and $p={ }_{\mu}$ proc (in $\vec{y}: \vec{\sigma}$; out $\vec{z}: \vec{\tau})\{s\}_{\vec{z}}$ :

$$
\begin{aligned}
p(\vec{e}, & \vec{r})^{\star}\left[\vec{v}^{\star} / \vec{x}\right] \\
& =\left(p^{\star} \vec{e}^{\star}\right)\left[\vec{v}^{\star} / \vec{x}\right] \\
& \left.=\left(\text { proc }(\text { in } \vec{y}: \vec{\sigma} ; \text { out } \vec{z}: \vec{\tau})\{s\}_{\vec{z}}\right)^{\star} \vec{w}^{\star}\right)\left[\vec{v}^{\star} / \vec{x}\right] \\
& \left.=\left(\text { fn }\left(\vec{y}: \vec{\sigma}^{\star}\right) \Rightarrow\{s\}^{\star}\left[\delta\left(\vec{\tau}^{\star}\right) / \vec{z}\right]\right) \vec{w}^{\star}\right)\left[\vec{v}^{\star} / \vec{x}\right] \\
& \rightsquigarrow\{s\}_{\vec{z}}^{\star}\left[\delta\left(\vec{\tau}^{\star}\right) / \vec{z}\right]\left[\vec{w}^{\star} / \vec{y}\right]\left[\vec{v}^{\star} / \vec{x}\right] \\
& =\{s[\vec{z} \hookleftarrow \vec{r}]\}_{\vec{r}}^{\star}\left[\delta\left(\vec{\tau}^{\star}\right) / \vec{r}\right]\left[\vec{w}^{\star} / \vec{y}\right]\left[\vec{v}^{\star} / \vec{x}\right] \text { by Lemma } 4.9 \\
& =\{s[\vec{z} \hookleftarrow \vec{r}]\}_{\vec{r}}^{\star}\left[\vec{w}^{\star} / \vec{y}\right]\left[\epsilon(\vec{\tau})^{\star} / \vec{r}\right]\left[\vec{v}^{\star} / \vec{x}\right] \text { by Lemma } 4.6 \\
& =\{s[\vec{z} \hookleftarrow \vec{r}][\vec{y} \leftarrow \vec{w}]\}_{\vec{r}}^{\star}\left[\epsilon(\vec{\tau})^{\star} / \vec{r}\right]\left[\vec{v}^{\star} / \vec{x}\right] \text { by Lemma } 4.10 \\
& =\{s[\vec{z} \hookleftarrow \vec{r}][\vec{y} \leftarrow \vec{w}]\}_{\vec{r}}^{\star}\left[\vec{v}^{\prime \star} / \vec{x}\right] \text { since } \vec{r} \subseteq \vec{x} .
\end{aligned}
$$


- $\quad($ S.CST)

$$
(\text { cst } y=e ; s)_{z}^{\star}\left[\vec{v}^{\star} / \vec{x}\right] \rightsquigarrow(s[y \leftarrow w])_{z}^{\star}\left[\vec{v}^{\star} / \vec{x}\right]
$$

Since $e={ }_{\mu} w$, by Lemma 4.11:

$$
\begin{aligned}
(\text { cst } & y=e ; s)^{\star}\left[\vec{v}^{\star} / \vec{x}\right] \\
& =\left(\text { let } y=e^{\star} \text { in }(s)^{\star}\right)\left[\vec{v}^{\star} / \vec{x}\right] \\
& =\left(\text { let } y=w^{\star} \text { in }(s)^{\star}\right)\left[\vec{v}^{\star} / \vec{x}\right] \\
& \rightsquigarrow\left((s)_{\vec{z}}^{\star}\left[w^{\star} / y\right]\right)\left[\vec{v}^{\star} / \vec{x}\right] \\
& =(s[y \leftarrow w])^{\star}\left[\vec{v}^{\star} / \vec{x}\right] \text { by Lemma } 4.10
\end{aligned}
$$

- $\quad($ S.FOR-I)

$$
\left(\text { for } y:=1 \text { to } e\{s\}_{\vec{z}}\right)^{\star}\left[\vec{v}^{\star} / \vec{x}\right] \rightsquigarrow\{\}_{z}^{\star}\left[\vec{v}^{\star} / \vec{x}\right]
$$

Since $e={ }_{\mu} 0$, by Lemma 4.11:

$$
\begin{aligned}
(\text { for } & \left.y:=1 \text { to } e\{s\}_{\vec{z}}\right)^{\star}\left[\vec{v}^{\star} / \vec{x}\right] \\
& =\operatorname{rec}\left(e^{\star}, \vec{z}, \lambda y \cdot \lambda \vec{z} \cdot\{s\}^{\star}\right)\left[\vec{v}^{\star} / \vec{x}\right] \\
& =\operatorname{rec}\left(0, \vec{z}, \lambda y \cdot \lambda \vec{z} \cdot\{s\}_{\vec{z}}^{\star}\right)\left[\vec{v}^{\star} / \vec{x}\right] \\
& \rightsquigarrow \vec{z}\left[\vec{v}^{\star} / \vec{x}\right] \\
& =\{\}_{\vec{z}}^{\star}\left[\vec{v}^{\star} / \vec{x}\right]
\end{aligned}
$$

- $\quad($ S.FOR-II)

$$
\left(\text { for } y:=1 \text { to } e\{s\}_{\vec{z}}^{\star}\right)\left[\vec{v}^{\star} / \vec{x}\right] \rightsquigarrow\left\{\text { for } y:=1 \text { to } \bar{q}\{s\}_{\vec{z}} ; s[y \leftarrow \overline{q+1}]\right\}_{\vec{z}}^{\star}\left[\vec{v}^{\star} / \vec{x}\right]
$$

Since $e={ }_{\mu} \overline{q+1}$, by Lemma 4.11:

$$
\begin{aligned}
\text { (for } & \left.y:=1 \text { to } e\{s\}_{\vec{z}}\right)^{\star}\left[\vec{v}^{\star} / \vec{x}\right] \\
& =\operatorname{rec}\left(e^{\star}, \vec{z}, \lambda y \cdot \lambda \vec{z} \cdot\{s\}_{\vec{z}}^{\star}\right)\left[\vec{v}^{\star} / \vec{x}\right] \\
& =\operatorname{rec}\left(S^{q+1}(0), \vec{z}, \lambda y \cdot \lambda \vec{z} \cdot\{s\}^{\star}\right)\left[\vec{v}^{\star} / \vec{x}\right] \\
& \rightsquigarrow\left(\left(\lambda \vec{z} \cdot\{s\}_{\vec{z}}^{\star}\left[S^{q+1}(0) / y\right]\right) \operatorname{rec}\left(S^{q}(0), \vec{z}, \lambda y \cdot \lambda \vec{z} \cdot\{s\}_{\vec{z}}^{\star}\right)\right)\left[\vec{v}^{\star} / \vec{x}\right] \\
& =\left(\operatorname{let} \vec{z}=\operatorname{rec}\left(S^{q}(0), \vec{z}, \lambda y \cdot \lambda \vec{z} \cdot\{s\}_{\vec{x}}^{\star}\right) \text { in }\{s[y \leftarrow \overline{q+1}]\}_{\vec{z}}^{\star}\right)\left[\vec{v}^{\star} / \vec{x}\right] \\
& =\left(\operatorname{let} \vec{z}=\left(\text { for } y:=1 \text { to } \bar{q}\{s\}_{\vec{z}}\right)^{\star} \text { in }\{s[y \leftarrow \overline{q+1}]\}_{\vec{z}}^{\star}\right)\left[\vec{v}^{\star} / \vec{x}\right] \\
& =\left\{\text { for } y:=1 \text { to } \bar{q}\{s\}_{\vec{z}} ; s[y \leftarrow q+1]\right\}_{\vec{z}}^{\star}\left[\vec{v}^{\star} / \vec{x}\right]
\end{aligned}
$$

Corollary 4.13. All programs of $\mathrm{LOOP}^{\omega}$ are terminating.

Proof. Indeed, since System $\mathrm{T}$ is strongly normalizing (see [Girard et al., 1989] for instance).

Let us now focus on the imperative counterpart of the hierarchy of fragment $T_{n}$ of Gödel System T. We define first the level of an imperative type and then the "iteration rank" of a Loop ${ }^{\omega}$ program as follows:

Definition 4.14. The level $\ell(\tau)$ of an imperative type $\tau$ is defined inductively by:

$-\quad \ell($ int $)=0$

- $\quad \ell\left(\right.$ proc $($ in $\vec{y}: \vec{\sigma} ;$ out $\left.\vec{z}: \vec{\tau})\{s\}_{\vec{z}}\right)=\max (\ell(\vec{\sigma})+1, \ell(\vec{\tau}))$ where $\ell(\vec{\tau})=\max \left(\ell\left(\tau_{1}\right), \ldots, \ell\left(\tau_{n}\right)\right)$.

Definition 4.15. We call "iteration rank" of a Loop ${ }^{\omega}$ program $p$ the maximum level of the types of mutable variables which annotate a loop of $p$.

Definition 4.16. The fragment $\mathrm{LOOP}^{n}$ of $\mathrm{LOOP}^{\omega}$ is defined as the set of programs with iteration rank less than $n$. 
Lemma 4.17. For any imperative types $\vec{\tau}, \partial\left(\left(\vec{\tau}^{\star}\right)^{\times}\right)=\ell(\vec{\tau})$.

Proposition 4.18. For any $\mathrm{LoOP}^{\omega}$ program $p$, the iteration rank of $p$ is the same as the recursion rank of $p^{\star}$.

Proof. Indeed, since for any loop which occurs in $p$ with mutable variables $\vec{z}: \vec{\tau}$ we have:

$$
\left(\text { for } y:=1 \text { to } e\{s\}_{\vec{z}}\right)^{\star}=\operatorname{rec}\left(e^{\star}, \vec{z}, \lambda y \cdot \lambda \vec{z} \cdot\{s\}_{\vec{z}}^{\star}\right)
$$

where this recursor has type $\left(\vec{\tau}^{\star}\right)^{\times}$and $\partial\left(\left(\vec{\tau}^{\star}\right)^{\times}\right)=\ell(\vec{\tau})$ by lemma 4.17 .

Remark 4.19. A function computable in the fragment LooP $^{0}$ is thus computable in $\mathrm{T}_{0}$, hence it is primitive recursive. Consequently, adding only higher-order procedures (without procedural variables) to the Loop language does not increase its expressive power.

In the next section, we shall be interested in $\mathrm{LOOP}^{\omega}$ programs which are translated into terms which contain no pairing and no pattern matching. The following lemma is straightforward (by definition of the translation).

Definition 4.20. We call "singular" a LoOP" program in which all procedures have exactly one in parameter and one out parameter and all blocks are annotated by exactly one mutable variable.

Lemma 4.21. For any singular Loop ${ }^{\omega}$ program $p$, the term $p^{\star}$ contains no product types (no tuples and no pattern matching).

\section{Expressiveness}

In this section, we show how to represent any functional of Gödel's system $\mathrm{T}$ by some LoOP $^{\omega}$ program. However, since there is no direct counterpart to pattern-matching in $\mathrm{LOOP}^{\omega}$, we consider in this section a variant of System $\mathrm{T}$ with explicit pairing function and projections. On the other hand, it is straightforward to encode a (lazy) pair as an anonymous procedure with no input parameter (a thunk).

Definition 5.1. We define $(\sigma * \tau)$ as unit $\rightarrow(\sigma \times \tau)$ and call $\mathcal{S}$ the set of types built inductively from int using the type constructors $\rightarrow$ and $*$.

Definition 5.2. For any terms $t: \sigma, u: \tau$ we write $\langle t, u\rangle: \sigma * \tau$ as an abbreviation for the term $\mathbf{f n}() \Rightarrow(t, u)$. Moreover, we define $\pi_{\sigma, \tau}^{1}: \sigma * \tau \rightarrow \sigma$ and $\pi_{\sigma, \tau}^{2}: \sigma * \tau \rightarrow \tau$ as the following abbreviations:

$$
\pi_{\sigma, \tau}^{1}(z)=\operatorname{let}(x, y)=z() \text { in } x \quad \text { and } \quad \pi_{\sigma, \tau}^{2}(z)=\operatorname{let}(x, y)=z() \text { in } y
$$

Lemma 5.3. The following reduction rules are derivable (where $v, w$ are values):

$$
\pi_{\sigma, \tau}^{1}<v, w>\rightsquigarrow^{\star} v \quad \pi_{\sigma, \tau}^{2}<v, w>\rightsquigarrow^{\star} w
$$

Definition 5.4. For any type $\sigma \in \mathcal{S}$ the translation $\sigma^{\diamond}$ is defined as follows:

$-\quad$ int $\diamond=i n t$

- $(\sigma \rightarrow \tau)^{\diamond}=\operatorname{proc}\left(\right.$ in $\sigma^{\diamond}$; out $\left.\tau^{\diamond}\right)$

$-\quad(\sigma * \tau)^{\diamond}=\operatorname{proc}\left(\right.$ out $\left.\sigma^{\diamond}, \tau^{\diamond}\right)$

Lemma 5.5. For any type $\sigma \in \mathcal{S}$, we have $\left(\sigma^{\diamond}\right)^{\star}=\sigma$. 
We are now ready to translate terms of System $\mathrm{T}$ into LoOP ${ }^{\omega}$ programs. More precisely, a term $t$ of type $\sigma$ is translated into a command $c$ with exactly one free mutable $r$ variable of type $\sigma^{\diamond}$ for the result (i.e., $\mathcal{O}(c)=r$ ). For simplicity, we shall define the translation only for terms that obey some syntactic criterion:

Definition 5.6. We call $\mathcal{V}$ and $\mathcal{L}$ the subset of values and terms of System $T$ defined by the following syntax:

$$
\begin{aligned}
v, w::= & x\left|S^{n}(0)\right| \lambda x . t \mid<t, u> \\
t, u::= & v|\operatorname{succ}(v)| \operatorname{pred}(v) \mid \operatorname{rec}(v, u, \lambda y . \lambda z . t) \\
& |(v w)|(t v)|(v u)| \pi_{\sigma, \tau}^{1}(v) \mid \pi_{\sigma, \tau}^{2}(v)
\end{aligned}
$$

Remark 5.7. Clearly, terms of $\mathcal{L}$ can be seen as terms of System $\mathrm{T}$ since succ, $\pi_{\sigma, \tau}^{1}$, $\pi_{\sigma, \tau}^{2}$ and $\langle t, u\rangle$ are just macro-definitions. Conversely, any term of System $\mathrm{T}$ (as defined in figure 2.1) is clearly equivalent to a term of $\mathcal{L}$. Indeed, the requirements that $v$ be a value in any sub-term of the form $\pi_{\sigma, \tau}^{1}(v), \pi_{\sigma, \tau}^{2}(v)$ or $\operatorname{rec}(v, u, \lambda y . \lambda z . t)$ and that either $t$ or $u$ be a value in an application $(t u)$ are easily fulfilled by naming intermediate results when necessary (using a $\beta$-redex to simulate a let).

Definition 5.8. For any value $v$ in $\mathcal{V}$ and any term $t$ in $\mathcal{L}$ such that $\Gamma \vdash t$ : $\sigma$, the translation $v^{\diamond}$ and $t_{r}^{\diamond}$ where $r$ is a fresh variable of type $\sigma^{\diamond}$ are defined by mutual induction as follows (the types of the terms are written as superscript when required):

$$
\begin{array}{ll}
- & S^{n}(0)^{\diamond}=\bar{n} \\
- & y^{\diamond}=y \\
- & \left(\text { fn } x: \tau \Rightarrow t^{\omega}\right)^{\diamond}=\operatorname{proc}\left(\text { in } x: \tau^{\diamond} ; \text { out } y: \omega^{\diamond}\right) t_{y}^{\diamond} \\
- & (<t, u>)^{\diamond}=\operatorname{proc}\left(\text { out } x: \sigma^{\diamond}, y: \tau^{\diamond}\right)\left\{t_{x}^{\diamond} ; u_{y}^{\diamond}\right\}_{x, y} \\
- & (v)_{r}^{\diamond}=r:=v^{\diamond} \\
- & \operatorname{succ}(v)_{r}^{\diamond}=\left\{r:=v^{\diamond} ; \operatorname{inc}(r)\right\}_{r} \\
- & \operatorname{pred}(v)_{r}^{\diamond}=\left\{r:=v^{\diamond} ; \operatorname{dec}(r)\right\}_{r} \\
- & \operatorname{rec}(v, u, \lambda y \cdot \lambda z \cdot t)_{r}^{\diamond}=\left\{u_{r}^{\diamond} ; \text { for } y:=1 \text { to } v^{\diamond}\left\{\operatorname{cst} z=r ; t_{r}^{\diamond}\right\}_{r}\right\}_{r} \\
- & (v w)_{r}^{\diamond}=v^{\diamond}\left(w^{\diamond} ; r\right) \\
- & \left(v u^{\tau}\right)_{r}^{\diamond}=\left\{\operatorname{var} y: \tau^{\diamond} ; u_{y}^{\diamond} ; v^{\diamond}(y ; r)\right\}_{r} \\
- & \left(t^{\tau \rightarrow \omega} v\right)_{r}^{\diamond}=\left\{\operatorname{var} x:(\tau \rightarrow \omega)^{\diamond} ; t_{x}^{\diamond} ; x\left(v^{\diamond} ; r\right)\right\}_{r} \\
- & \pi_{\sigma, \tau}^{1}(v)_{r}^{\diamond}=\left\{\operatorname{var} y: \tau^{\diamond} ; v^{\diamond}(r, y)\right\}_{r} \\
- & \pi_{\sigma, \tau}^{2}(v)_{r}^{\diamond}=\left\{\operatorname{var} x: \sigma^{\diamond} ; v^{\diamond}(x, r)\right\}_{r}
\end{array}
$$

Remark 5.9. In Definition 5.8, we tried to reach a compromise between the simplicity of the translation and the effort required to prepare the term before its translation. It is however possible to generalize the above translation to arbitrary terms of System $\mathrm{T}$ (without restricting them to $\mathcal{L}$ ). For instance, $\operatorname{rec}(e, u, \lambda z . \lambda y . t)_{r}^{\diamond}$ where $e$ is an arbitrary terms (not necessarily a value) can be translated as follows:

$$
\operatorname{rec}(e, u, \lambda z \cdot \lambda y \cdot t)_{r}^{\diamond}=\left\{u_{r}^{\diamond} ; \operatorname{var} n: i n t ; e_{n}^{\diamond} ; \text { for } y:=1 \text { to } n\left\{\operatorname{cst} z=r ; t_{r}^{\diamond}\right\}_{r}\right\}_{r}
$$

Example 5.10. Recall the definition of the Ackermann function given in Section 2:

$$
a c k(n, m)=(\operatorname{rec}(m, \lambda y \cdot \operatorname{succ}(y), \lambda h . \lambda i . \lambda y \cdot \operatorname{rec}(y,(h S(0)), \lambda k . \lambda j .(h k))) n)
$$

By applying the translation with variable $r$ : int for the result, we get the following block for $\operatorname{ack}(n, m)_{r}^{\diamond}$ : 


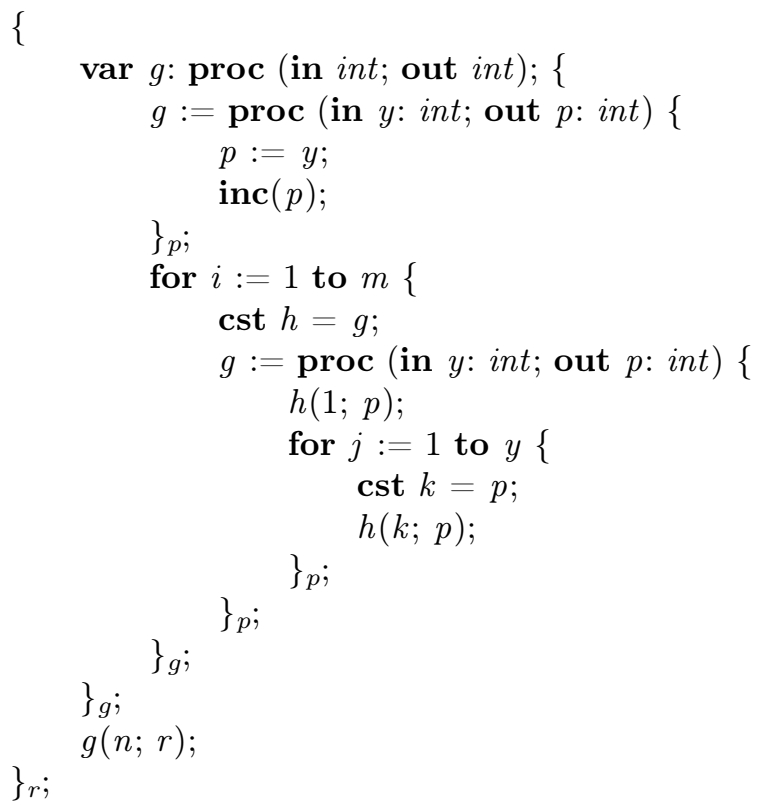

We obtain thus essentially the body of the imperative version of the Ackermann function given in Section 2.1 (apart from non-mandatory blocks and constant declarations).

Note that all identifiers of the source term are mapped to read-only variables (hence the introduction of a constant declaration in the translation of a recursor). This property ensures that mutable variables do not occur in the body of procedures in the resulting Loop $^{\omega}$ program: the only mutable variables are fresh variables introduced during the translation.

Lemma 5.11. Given a term $t \in \mathcal{L}$ such that $\Gamma \vdash t: \sigma$ and a fresh mutable variable $r$ of type $\sigma^{\diamond}$ we have $\Gamma^{\diamond} ; r: \sigma^{\diamond} \vdash t_{r}^{\diamond}$ : comm.

Proof. By induction on $t$.

For any term $t \in \mathcal{L}$, the fresh mutable variable $r$ in $t_{r}^{\diamond}$ is always initialized before it is read, and thus $r$ is not free in $\left(t_{r}^{\diamond}\right)^{\star}$. This property is stated more formally by the following lemma.

Lemma 5.12. Given a term $t \in \mathcal{L}$ such that $\Gamma \vdash t: \sigma$ and a fresh mutable variable $r$ of type $\sigma^{\diamond}$ we have $r \notin \operatorname{FId}\left(\left(t_{r}^{\diamond}\right)^{\star}\right)$.

Proof. By induction on $t$.

Let us write $\rightarrow$ for the reflexive, transitive and contextual closure (with respect to arbitrary contexts) of the following general $\beta \pi$-reduction rule (where $t_{1}, \ldots, t_{n}$ are not necessarily values):

$$
\text { let }\left(x_{1}, \ldots, x_{n}\right)=\left(t_{1}, \ldots, t_{n}\right) \text { in } u \mapsto u\left[t_{1} / x_{1}, \ldots, t_{n} / x_{n}\right]
$$

As expected, the translated $\left(t_{r}^{\diamond}\right)^{\star}$ of a term $t$ requires several "administrative" reductions in order to recover the original term.

Theorem 5.13. Given a term $t \in \mathcal{L}$ such that $\Gamma \vdash t: \sigma$ and a fresh mutable variable $r$ of type $\sigma^{\diamond}$ we have $\left(t_{r}^{\diamond}\right)^{\star} \rightarrow t$.

Proof. By mutual induction, we prove that for any value $v \in \mathcal{V}$ such that $\Gamma \vdash v$ : $\tau$ we have $\left(v^{\diamond}\right)^{\star} \rightarrow v$ and for any term $t \in \mathcal{L}$ such that $\Gamma \vdash t: \sigma$ and any fresh mutable variable $r$ of type $\sigma^{\diamond}$ we have $\left(t_{r}^{\diamond}\right)^{\star} \rightarrow t$.

- $\left(S^{n}(0)^{\diamond}\right)^{\star}=\bar{n}^{\star}=S^{n}(0)$ 
- $\left(y^{\diamond}\right)^{\star}=y^{\star}=y$

- $\quad\left(\left(\mathbf{f n} x: \tau \Rightarrow t^{\omega}\right)^{\diamond}\right)^{\star}$

$=\left(\operatorname{proc}\left(\text { in } x: \tau^{\diamond} ; \text { out } y: \omega^{\diamond}\right) t_{y}^{\diamond}\right)^{\star}$

$=\mathbf{f n}(x: \tau) \Rightarrow\left(t_{y}^{\diamond}\right)^{\star}[\delta(\omega) / y]$

$=\mathbf{f n}(x: \tau) \Rightarrow\left(t_{y}^{\diamond}\right)^{\star}$ since $y \notin \operatorname{FId}\left(\left(t_{y}^{\diamond}\right)^{\star}\right)$

$\rightarrow \mathbf{f n} x: \tau \Rightarrow t$ by induction hypothesis.

- $\left(<t, u>_{r}^{\diamond}\right)^{\star}$

$=\left(\operatorname{proc}\left(\text { out } x: \sigma^{\diamond}, y: \tau^{\diamond}\right)\left\{t_{x}^{\diamond} ; u_{y}^{\diamond}\right\}_{x, y}\right)^{\star}$

$=\left(\mathbf{f n}() \Rightarrow\right.$ let $x=\left(t_{x}^{\diamond}\right)^{\star}$ in let $y=\left(u_{y}^{\diamond}\right)^{\star}$ in $\left.(x, y)\right)$

$\rightarrow \mathbf{f n}() \Rightarrow\left(\left(t_{x}^{\diamond}\right)^{\star},\left(u_{y}^{\diamond}\right)^{\star}\right)$

$\rightarrow\langle t, u\rangle$ by induction hypothesis.

- $\quad\left((v)_{r}^{\diamond}\right)^{\star}$

$=\left(r:=v^{\diamond}\right)^{\star}$

$=\left(v^{\diamond}\right)^{\star}$

$\rightarrow v$ by induction hypothesis.

- $\quad\left(\operatorname{succ}(v)_{r}^{\diamond}\right)^{\star}$

$=\left\{r:=v^{\diamond} ; \operatorname{inc}(r)\right\}_{r}^{\star}$

$=$ let $r=\left(v^{\diamond}\right)^{\star}$ in let $r=\operatorname{succ}(r)$ in $r$

$\rightarrow$ let $r=\operatorname{succ}\left(\left(v^{\diamond}\right)^{\star}\right)$ in $r$

$\rightarrow \operatorname{succ}\left(\left(v^{\diamond}\right)^{\star}\right)$

$\rightarrow \operatorname{succ}(v)$ by induction hypothesis.

- The case of pred is similar to succ.

- $\quad\left(\operatorname{rec}(v, u, \lambda y \cdot \lambda z . t)_{r}^{\diamond}\right)^{\star}$

$=\left\{u_{r}^{\diamond} ; \text { for } y:=1 \text { to } v^{\diamond}\left\{\mathbf{c s t} z=r ; t_{r}^{\diamond}\right\}_{r}\right\}_{r}^{\star}$

$=$ let $r=\left(u_{r}^{\diamond}\right)^{\star}$ in let $r=\operatorname{rec}\left(\left(v^{\diamond}\right)^{\star}, r, \lambda y \cdot \lambda r\right.$.let $z=r$ in $\left.\left(t_{r}^{\diamond}\right)^{\star}\right)$ in $r$

$\rightarrow$ let $r=\operatorname{rec}\left(\left(v^{\diamond}\right)^{\star},\left(u_{r}^{\diamond}\right)^{\star}, \lambda y \cdot \lambda z \cdot\right.$ let $z=z$ in $\left.\left(t_{r}^{\diamond}\right)^{\star}\right)$ in $r$ since $r \notin F I d\left(\left(t_{r}^{\diamond}\right)^{\star}\right)$

$\rightarrow \operatorname{rec}\left(\left(v^{\diamond}\right)^{\star},\left(u_{r}^{\diamond}\right)^{\star}, x, \lambda z \cdot \lambda y \cdot\left(t_{r}^{\diamond}\right)^{\star}\right)$

$\rightarrow \operatorname{rec}(v, u, \lambda z . \lambda y . t)$ by induction hypothesis.

- $\left(\left(v^{\tau \rightarrow \sigma} w\right)_{r}^{\diamond}\right)^{\star}$

$=\left(v^{\diamond}\left(w^{\diamond} ; r\right)\right)^{\star}$

$=\left(v^{\diamond}\right)^{\star}\left(w^{\diamond}\right)^{\star}$

$\rightarrow(v w)$ by induction hypothesis.

- $\left(\left(t^{\tau \rightarrow \sigma} v\right)_{r}^{\diamond}\right)^{\star}$

$=\left\{\operatorname{var} p:(\tau \rightarrow \sigma)^{\diamond} ; t_{p}^{\diamond} ; p\left(v^{\diamond} ; r\right)\right\}_{r}^{\star}$

$=\left(\right.$ let $p=\left(t_{p}^{\diamond}\right)^{\star}$ in let $r=p\left(v^{\diamond}\right)^{\star}$ in $\left.r\right)[\delta(\tau \rightarrow \sigma) / p]$

$=$ let $p=\left(t_{p}^{\diamond}\right)^{\star}$ in let $r=p\left(v^{\diamond}\right)^{\star}$ in $r$ since $p \notin \operatorname{FId}\left(\left(t_{p}^{\diamond}\right)^{\star}\right)$

$\rightarrow\left(t_{p}^{\diamond}\right)^{\star}\left(v^{\diamond}\right)^{\star}$

$\rightarrow(t v)$ by induction hypothesis.

- $\left(\left(v^{\tau \rightarrow \sigma} u\right)_{r}^{\diamond}\right)^{\star}$

$=\left\{\operatorname{var} y: \tau^{\diamond} ; u_{y}^{\diamond} ; v(y ; r)\right\}_{r}^{\star}$

$=\left(\right.$ let $y=\left(u_{y}^{\diamond}\right)^{\star}$ in let $r=\left(v^{\diamond}\right)^{\star} y$ in $\left.r\right)[\delta(\tau) / y]$

$=$ let $y=\left(u_{y}^{\diamond}\right)^{\star}$ in let $r=\left(v^{\diamond}\right)^{\star} y$ in $r$ since $y \notin \operatorname{FId}\left(\left(u_{y}^{\diamond}\right)^{\star}\right)$

$\rightarrow\left(v^{\diamond}\right)^{\star}\left(u_{y}^{\diamond}\right)^{\star}$

$\rightarrow(v u)$ by induction hypothesis.

- $\left(\pi_{\sigma, \tau}^{1}(v)_{r}^{\diamond}\right)^{\star}=$

$=\left\{\operatorname{var} y: \tau^{\diamond} ; v^{\diamond}(r, y)\right\}_{r}^{\star}$

$=\left(\right.$ let $(r, y)=\left(v^{\diamond}\right)^{\star}()$ in $\left.r\right)[\delta(\tau) / y]$

$=$ let $(r, y)=\left(v^{\diamond}\right)^{\star}()$ in $r$ since $y \notin \operatorname{FId}\left(\left(v^{\diamond}\right)^{\star}\right)$

$\rightarrow$ let $(r, y)=v()$ in $r$ by induction hypothesis.

$=\pi_{\sigma, \tau}^{1}(v)$ by definition of $\pi_{\sigma, \tau}^{1}$. 
- The case of $\pi_{\sigma, \tau}^{2}$ is similar to $\pi_{\sigma, \tau}^{1}$.

Lemma 5.14. For any functional type $\tau, \partial\left(\tau^{\diamond}\right)=\ell(\tau)$.

Proposition 5.15. Given a term $t \in \mathcal{L}$ such that $\Gamma \vdash t: \sigma$ and a fresh mutable variable $p$ of type $\sigma^{\diamond}$, the iteration rank of $t_{p}^{\diamond}$ is the same as the recursion rank of $t$.

Proof. Indeed, any occurrence of a recursor type $\tau$ in $t$ is translated as follows:

$$
\operatorname{rec}(x, u, \lambda y \cdot \lambda z \cdot s)_{r}^{\diamond}=\left\{u_{r}^{\diamond} ; \text { for } y:=1 \text { to } x\left\{\operatorname{cst} z=r ; s_{r}^{\diamond}\right\}_{r}\right\}_{r}
$$

where $r$ is a mutable variable of type $\tau^{\diamond}$. We can conclude since $r$ occurs inside a loop and $\partial\left(\tau^{\diamond}\right)=\ell(\tau)$ by lemma 5.14 .

Remark 5.16. Note that a term with no product types (no pairs and no projections) is translated into a singular $\mathrm{LOOP}^{\omega}$ program.

\section{Applications}

\subsection{Characterizing elementary functions}

A first application of the results of this paper is extensional: we give a new characterization of the class of Csillag-Kalmar elementary recursive functions (the class $\mathcal{E}_{3}$ in Grzegorczyk hierarchy). In [Beckmann and Weiermann, 2000], the authors consider a variant of System $\mathrm{T}$ with an explicit discriminator $\mathcal{D}$ and an iterator $\mathcal{I}$ instead of a recursor and they prove the following theorem:

Theorem 6.1. [Beckmann and Weiermann, 2000] The set of terms of System T which contain no $\lambda$-abstraction of the form $\lambda x \ldots \mathcal{I}(t, \ldots)$ where $x$ occurs in $t$ compute exactly the class of elementary recursive functions.

It is well-known that both operators $\mathcal{D}$ and $\mathcal{I}$ are special cases of the recursor where $\mathcal{D}(n, a, b) \equiv \operatorname{rec}(n, a, \lambda i . \lambda x . b)$ and $\mathcal{I}(n, b, \lambda y . a) \equiv \operatorname{rec}(n, b, \lambda i . \lambda y . a)$ where $x, i$ are fresh identifiers. On the imperative side, these operators correspond respectively to the standard compound statements if ... then ... else ... and loop $n\{\ldots\}$ (where there is no iteration variable available inside the body of the loop).

Given a term $t$, their restriction amounts to requiring that in any sub-term $\mathcal{I}(u, \ldots)$ of $t$ the free identifiers of $u$ are actually free identifiers of $t$ (input identifiers). Let us now define the imperative counterpart of this restriction.

Definition 6.2. We call "elementary" any singular LoOP ${ }^{\omega}$ program $p$ such that $\Gamma ; r$ : int $\vdash p$ : comm and any loop has the form $\left\{\operatorname{var} n \text { : int; }\left\{s_{1}\right\}_{n} ; \text { loop } n\left\{s_{2}\right\}_{z}\right\}_{z}$ where the only free read-only variables of $s_{1}$ are in $\Gamma$.

Remark 6.3. In this definition, the loop statement corresponds to a macro-definition for a for loop in which the iteration variable does not occur. Note also that if ... then ... else ... statements are allowed without restriction in $p$. In particular, we obtain that any Loop $^{\omega}$ program in which any bound of loop is a read-only input variable is elementary.

As a corollary of Theorems 4.12 and 5.13 we obtain the following result:

Corollary 6.4. The elementary singular Loop ${ }^{\omega}$ programs compute exactly the class of elementary recursive functions.

Proof. Indeed, let $p$ be and elementary singular Loop ${ }^{\omega}$ program. For any occurrence of a loop statement in $p$ we have: 


$$
\begin{aligned}
& \left\{\operatorname{var} n: \text { int; }\left\{s_{1}\right\}_{n} ; \text { loop } n\left\{s_{2}\right\}_{z}\right\}_{z}^{\star} \\
& \quad=\operatorname{let} n=\left\{s_{1}\right\}_{n}^{\star} \text { in let } z=\operatorname{rec}\left(n, z, \lambda z \cdot \lambda y \cdot\left\{s_{2}\right\}_{z}^{\star}\right) \operatorname{in} z \\
& \quad \rightarrow \operatorname{rec}\left(\left\{s_{1}\right\}_{n}^{\star}, z, \lambda y \cdot \lambda z \cdot\left\{s_{2}\right\}_{z}^{\star}\right)=\mathcal{I}\left(\left\{s_{1}\right\}_{n}^{\star}, z, \lambda z \cdot\left\{s_{2}\right\}_{z}^{\star}\right)
\end{aligned}
$$

Since the only free read-only variables of $\left\{s_{1}\right\}_{n}^{\star}$ are input variables, the term $p^{\star}$ computes thus an elementary function. Conversely, for any term $t: \sigma$ of System T which obeys Beckmann and Weirmann's restriction, the term $t_{r}^{\diamond}$ (where $r$ is a fresh mutable variable of type $\sigma^{\diamond}$ ) is an elementary singular LoOP $^{\omega}$ programs (see remark 5.9).

Remark 6.5. If $x$ is an identifier in $\operatorname{rec}\left(x, z, \lambda y \cdot \lambda z \cdot\{s\}_{z}^{\star}\right)$ there is no need for introducing the local variable $n$ in its imperative translation and we obtain thus the following simpler form loop $x\{s\}_{z}$. As a special case, we obtain thus that any Loop ${ }^{\omega}$ program in which any bound of a loop is an input read-only variable is elementary. Actually, we conjecture that such programs are sufficient to represent any elementary function (a proof of this result would follow step by step the proof given for System $\mathrm{T}$ in Section 4 of [Beckmann and Weiermann, 2000]).

Remark 6.6. For simplicity, we restricted ourselves to singular LoOP ${ }^{\omega}$ programs since [Beckmann and Weiermann, 2000] do not consider product types. However, since their result is extensional, any encoding of product types in System $\mathrm{T}$ (which does not use the recursor) would allow to generalize the above corollary to non-singular elementary Loop ${ }^{\omega}$ programs. Such an encoding is the following:

The product $\sigma \times \tau$ is defined by induction on $\sigma$ and then on $\tau$. Firstly, int $\times$ int is encoded as int $\rightarrow$ int where $\langle t, u\rangle_{\text {int } \times \text { int }} \equiv \lambda b . \mathcal{D}(b, t, u)$ and with $\pi_{\text {int } \times \text { int }}^{1}(p) \equiv(p 0)$ and $\pi_{\text {int } \times \text { int }}^{2}(p) \equiv(p S(0))$. Now int $\times(\sigma \rightarrow \varsigma)$ is defined as $\sigma \rightarrow($ int $\times \varsigma)$ and $(\sigma \rightarrow \varsigma) \times \tau$ as $\sigma \rightarrow(\varsigma \times \tau)$. The corresponding pairing functions and projections are summarized below. It is straightforward to prove that $\pi^{1}\langle t, u\rangle=t$ and $\pi^{2}\langle t, u\rangle=u$ for any types $\sigma, \tau$ (note however that the $\eta$-rule is required).

$$
\begin{aligned}
& \langle t, u\rangle_{\text {int } \times(\sigma \rightarrow \varsigma)}=\lambda x: \sigma \cdot\langle t,(u x)\rangle_{\text {int } \times \varsigma} \quad\langle t, u\rangle_{(\sigma \rightarrow \varsigma) \times \tau}=\lambda x: \sigma \cdot\langle(t x), u\rangle_{\varsigma \times \tau} \\
& \pi_{\text {int } \times(\sigma \rightarrow \varsigma)}^{1}(z)=\pi_{\text {int } \times \varsigma}^{1}(z \delta(\sigma)) \quad \pi_{(\sigma \rightarrow \varsigma) \times \tau}^{1}(z)=\lambda x: \sigma \cdot \pi_{\varsigma \times \tau}^{1}(z x) \\
& \pi_{\text {int } \times(\sigma \rightarrow \varsigma)}^{2}(z)=\lambda x: \sigma \cdot \pi_{i n t \times \varsigma}^{2}(z x) \quad \pi_{(\sigma \rightarrow \varsigma) \times \tau}^{2}(z)=\pi_{\varsigma \times \tau}^{2}(z \delta(\sigma))
\end{aligned}
$$

\subsection{The minimum problem}

The second application is intensional and is related to the so-called minimum problem. In [Colson and Fredholm, 1998], the authors proved that in call-by-value System T, any algorithm which computes a non-trivial binary function (where trivial means constant or projection plus constant), has a time-complexity which is at least linear in one of the inputs. As a consequence, they obtain the following result:

Theorem 6.7. [Colson and Fredholm, 1998] There is no term of call-by-value System T which computes the minimum of two natural numbers with time-complexity $O(\min (n, m))$.

Remark 6.8. It is worth noticing that this property depends strongly on the reduction rules used for the recursor (and not only on the strategy). For instance, the property does not hold if we consider the following reduction rule for rec (which is derivable in call-byname, but not in call-by-value):

$$
\operatorname{rec}(S(n), v, \lambda x . \lambda y . t) \rightsquigarrow t[n / x, \operatorname{rec}(n, b, \lambda x . \lambda y . t) / y]
$$

Indeed, since we consider weak reduction and since $y$ may occurs under the scope of a $\lambda$ abstraction in $t$, there no reason that $\operatorname{rec}(n, b, \lambda x$. $\lambda y . t)$ be the next redex to contract. In fact, one can easily show that call-by-name evaluation can be simulated under call-byvalue evaluation using this rule and the usual thunk-based encoding [Hatcliff and Danvy, 1997]. 
Note that [Colson and Fredholm, 1998] do not consider product types nor a predecessor operation. However, as a direct corollary of this theorem and the lock-step simulation, we can still obtain:

Corollary 6.9. There is no singular $\mathrm{LOOP}^{\omega}$ program without dec which computes the minimum of two natural numbers $n, m$ with time-complexity $O(\min (n, m))$.

In order to generalize this result to arbitrary $\operatorname{Loop}^{\omega}$ programs, we need to extend first Colson and Fredholm's theorem. Although their proof technique seems to apply to product types, we did not succeed in extending it with a one-step predecessor operation. However, we present a direct proof of the result for our variant of System $T$ in Appendix A and then we get the expected corollary.

Corollary 6.10. There is no LoOP ${ }^{\omega}$ program which computes the minimum of two natural numbers $n, m$ with time-complexity $O(\min (n, m))$.

\section{Appendix A Ultimate Obstinacy}

In this appendix, we show that (non-trivial) terms of System $\mathrm{T}$ are "ultimately obstinate" (this terminology is borrowed from [Colson, 1991]) even in the presence a one-step predecessor operation and product types. However, since the techniques developed in [Colson and Fredholm, 1998] do not seem to generalize easily to this case, we present here a direct proof of this result.

We first extend the syntax of terms and values with an infinite number of constants $\perp_{i}^{k}$ of type $N$ and we supplement our set of reduction rules with the following rule:

$$
C\left[\operatorname{pred}\left(\perp_{i}^{k}\right)\right] \rightsquigarrow C\left[\perp_{i}^{k+1}\right]
$$

We denote by $\rightsquigarrow_{\perp}$ this extended rewriting system. Intuitively, a constant $\perp_{i}^{k}$ represents a "sufficiently large" natural number (on which the predecessor can be applied at least $k$ times). The strong normalization of reduction $\rightsquigarrow_{\perp}^{*}$ for well-typed terms is a consequence of the following lemma (since the reduction $\rightsquigarrow$ is strongly normalizing for well-typed terms).

Lemma A.1. If $t\left[\perp_{1}^{k_{1}} / x_{1}, \ldots, \perp_{n}^{k_{n}} / x_{n}\right] \rightsquigarrow u\left[\perp_{1}^{k_{1}} / x_{1}, \ldots, \perp_{n}^{k_{n}} / x_{n}\right]$ for some terms $t, u$ then $t\left[0 / x_{1}, \ldots, 0 / x_{n}\right] \rightsquigarrow u\left[0 / x_{1}, \ldots, 0 / x_{n}\right]$.

Since terms now possibly contain constants $\perp_{i}^{k}$ of type $N$, even a well-typed term can get stuck during evaluation (the term cannot be evaluated further) although it is not yet a value. This lemma allows us to split the set of terms into constant, trivial and ultimately obstinate terms.

Lemma A.2. Given a well-typed term $t$ of type $N$ with free variables $x_{1}, \ldots, x_{n}$ of type $N$, if $t\left[\perp_{1}^{0} / x_{1}, \ldots, \perp_{n}^{0} / x_{n}\right] \rightsquigarrow_{\perp}^{p} v$ where $v$ is in normal form then one of the following cases holds:

- $v$ is a value $S^{k}(0)$ (we say that $t$ is "constant")

- $v$ is a value $S^{k}\left(\perp_{i}^{m}\right)$ with $m \leqslant p$ (we say that $t$ is "trivial")

- there is an evaluation context $C[]$ such that $v=C\left[\operatorname{rec}\left(\perp_{i}^{m}, v_{2}, v_{3}\right)\right]$ and $m \leqslant p$ (we say that $t$ is "ultimately obstinate")

Proof. By inspection of normal forms (and then by induction on the derivation to show that $m \leqslant p)$.

Definition A.3. The S-substitution $t \llbracket S\left(\perp_{i}\right) / \perp_{i} \rrbracket$ is the homomorphic extension of the basic function defined as follows:

$$
-\quad \perp_{i}^{0} \llbracket S\left(\perp_{i}\right) / \perp_{i} \rrbracket=S\left(\perp_{i}^{0}\right)
$$




$$
\text { - } \quad \perp_{i}^{k+1} \llbracket S\left(\perp_{i}\right) / \perp_{i} \rrbracket=\perp_{i}^{k}
$$

We write $t \llbracket S^{p}\left(\perp_{i}\right) / \perp_{i} \rrbracket$ for the generalized $S$-substitution defined by induction on $p$ with $t \llbracket S^{0}\left(\perp_{i}\right) / \perp_{i} \rrbracket=t$ and $t \llbracket S^{p+1}\left(\perp_{i}\right) / \perp_{i} \rrbracket=t \llbracket S\left(\perp_{i}\right) / \perp_{i} \rrbracket \llbracket S^{p}\left(\perp_{i}\right) / \perp_{i} \rrbracket$.

Lemma A.4. For any terms $t, u$, if $t \rightsquigarrow u$ then $t \llbracket S\left(\perp_{i}\right) / \perp_{i} \rrbracket \rightsquigarrow u \llbracket S\left(\perp_{i}\right) / \perp_{i} \rrbracket$

Proof. Check that S-substitution commutes with the reduction rules given in Figure 2.1 and with the rule $C\left[\operatorname{pred}\left(\perp_{i}^{k}\right)\right] \rightsquigarrow C\left[\perp_{i}^{k+1}\right]$.

The following lemma (together with Lemma A.1) allows us to apply Lemma A.2 on genuine natural numbers.

Lemma A.5. Given a well-typed term $t$ with constants $\perp_{1}^{0}, \ldots, \perp_{n}^{0}$ of type $N$, if $t \rightsquigarrow \perp v$ then $t \llbracket S^{p}\left(\perp_{1}\right) / \perp_{1}, \ldots, S^{p}\left(\perp_{n}\right) / \perp_{n} \rrbracket \rightsquigarrow \perp v \llbracket S^{p}\left(\perp_{1}\right) / \perp_{1}, \ldots, S^{p}\left(\perp_{n}\right) / \perp_{n} \rrbracket$

Proof. Apply Lemma A.4 repeatedly $p$ times for each $\perp_{i}$.

Lemma A.6. (CONSTANT TERMS) Given a well-typed constant term $t: N$ with free variables $x_{1}, \ldots, x_{n}$ of type $N$, there exist $k, p \in \mathbb{N}$ such that for any $p_{1} \geqslant 0, \ldots, p_{n} \geqslant 0, t\left[S^{p_{1}}(0) /\right.$ $\left.x_{1}, \ldots, S^{p_{n}}(0) / x_{n}\right] \rightsquigarrow^{p} S^{k}(0)$.

Proof. Let $t^{\prime}=t\left[\perp_{1}^{0} / x_{1}, \ldots, \perp_{n}^{0} / x_{n}\right]$ and $p, k$ be such that $t^{\prime} \rightsquigarrow^{p} S^{k}(0)$ by Lemma A.2. Since by Lemma A.5, $t^{\prime} \llbracket S^{p_{1}}\left(\perp_{1}\right) / \perp_{1}, \ldots, S^{p_{n}}\left(\perp_{n}\right) / \perp_{n} \rrbracket \rightsquigarrow{ }_{\perp}^{p} S^{k}(0)$ and since $t\left[S^{p_{1}}\left(\perp_{1}^{0}\right) / x_{1}, \ldots\right.$, $S^{p_{n}}\left(\perp_{n}^{0}\right) / x_{n} \rrbracket=t^{\prime} \llbracket S^{p_{1}}\left(\perp_{1}\right) / \perp_{1}, \ldots, S^{p_{n}}\left(\perp_{n}\right) / \perp_{n} \rrbracket$ we have $t^{\prime} \llbracket S^{p_{1}}\left(\perp_{1}\right) / \perp_{1}, \ldots, S^{p_{n}}\left(\perp_{n}\right) /$ $\perp_{n} \rrbracket \rightsquigarrow{ }_{\perp}^{p} S^{k}(0)$. We conclude by Lemma A.1 that $t\left[S^{p_{1}}(0) / x_{1}, \ldots, S^{p_{n}}(0) / x_{n}\right] \rightsquigarrow^{p} S^{k}(0)$.

Lemma A.7. (TRIVIAL TERMS) Given a well-typed trivial term $t: N$ with free variables $x_{1}, \ldots, x_{n}$ of type $N$, there exist $i, m, p, k$ with $1 \leqslant i \leqslant n$ and $m \leqslant p$ such that for any $p_{1} \geqslant$ $0, \ldots, p_{n} \geqslant 0, t\left[S^{p_{1}}(0) / x_{1}, \ldots, S^{p_{n}}(0) / x_{n}\right] \rightsquigarrow^{p} S^{p_{i} \dot{-} m+k}(0)$.

Proof. Let $t^{\prime}=t\left[\perp_{1}^{0} / x_{1}, \ldots, \perp_{n}^{0} / x_{n}\right]$ and $p, k, m$ be such that $t^{\prime} \rightsquigarrow^{p} S^{k}\left(\perp_{i}^{m}\right)$ by Lemma A.2. By Lemma A.5, $t^{\prime} \llbracket S^{p_{1}}\left(\perp_{1}\right) / \perp_{1}, \ldots, S^{p_{n}}\left(\perp_{n}\right) / \perp_{n} \rrbracket \rightsquigarrow{ }_{\perp}^{p} S^{k}\left(S^{p_{i}-m}\left(\perp_{i}^{0}\right)\right)$ if $p_{i} \geqslant m$, and $t^{\prime} \llbracket S^{p_{1}}\left(\perp_{1}\right) / \perp_{1}, \ldots, S^{p_{n}}\left(\perp_{n}\right) / \perp_{n} \rrbracket \rightsquigarrow{ }_{\perp}^{p} S^{k}\left(\perp_{i}^{m-p_{i}}\right)$ if $p_{i}<m$. Since $t\left[S^{p_{1}}\left(\perp_{1}^{0}\right) / x_{1}, \ldots, S^{p_{n}}\left(\perp_{n}^{0}\right) /\right.$ $\left.x_{n}\right]=t^{\prime} \llbracket S^{p_{1}}\left(\perp_{1}\right) / \perp_{1}, \ldots, S^{p_{n}}\left(\perp_{n}\right) / \perp_{n} \rrbracket$, we conclude by Lemma A.1 that $t\left[S^{p_{1}}(0) / x_{1}, \ldots\right.$, $\left.S^{p_{n}}(0) / x_{n}\right] \rightsquigarrow{ }^{p} S^{k}\left(S^{p_{i}-m}(0)\right)$.

Remark A.8. Clearly, trivial terms compute only functions definable (with the usual mathematical notation) as $\left(x_{1}, \ldots, x_{n}\right) \mapsto\left(x_{i} \dot{-} m\right)+k$ (for some constants $m, k$ ).

Lemma A.9. Given an evaluation context $C[]$, if $C\left[\operatorname{rec}\left(S^{k}(0), v_{2}, v_{3}\right)\right]$ is well-typed and $k>0$ then $C\left[\operatorname{rec}\left(S^{k}(0), v_{2}, v_{3}\right)\right] \rightsquigarrow C^{\prime}\left[\operatorname{rec}\left(S^{k-1}(0), v_{2}, v_{3}\right)\right]$ where $C^{\prime}[]$ is again an evaluation context.

Proof. Indeed, $C^{\prime}=C\left[\left(v_{3}[] S^{k}(0)\right)\right]$.

Theorem A.10. (Ultimately obStinate terms) Given an ultimately obstinate term $t$ of type $N$ with free variables $x_{1}, \ldots, x_{n}$ of type $N$, there exist $i, m \in \mathbb{N}$ with $1 \leqslant i \leqslant n$ such that for any $p_{1} \geqslant p, \ldots, p_{n} \geqslant p, t\left[S^{p_{1}}(0) / x_{1}, \ldots, S^{p_{n}}(0) / x_{n}\right]$ reaches its normal form in at least $p_{i}$ reductions steps.

Proof. If $t^{\prime}=t\left[\perp_{1}^{0} / x_{1} \ldots, \perp_{n}^{0} / x_{n}\right]$, by Lemma A.2, $t^{\prime} \stackrel{\perp}{\perp}^{p} C\left[\operatorname{rec}\left(\perp_{i}^{m}, v_{2}, v_{3}\right)\right]$ for some $p, m$. Now, $t\left[S^{p_{1}}\left(\perp_{1}^{0}\right) / x_{1} \ldots, S^{p_{n}}\left(\perp_{n}^{0}\right) / x_{n}\right]=t^{\prime} \llbracket S^{p_{1}}\left(\perp_{1}\right) / \perp_{1} \ldots, S^{p_{n}}\left(\perp_{n}\right) / \perp_{n} \rrbracket$ and since $p_{i} \geqslant m$, $t^{\prime} \llbracket S^{p_{1}}\left(\perp_{1}\right) / \perp_{1} \ldots, S^{p_{n}}\left(\perp_{n}\right) / \perp_{n} \rrbracket \rightsquigarrow{ }_{\perp}^{p} C\left[\operatorname{rec}\left(\perp_{i}^{p_{i}-m}, v_{2}, v_{3}\right)\right]$ by Lemma A.5. We have then $t\left[S^{p_{1}}(0) / x_{1} \ldots, \quad S^{p_{n}}(0) / x_{n}\right] \quad \rightsquigarrow p \quad C\left[\operatorname{rec}\left(S^{p_{i}-m}(0), \quad v_{2}, \quad v_{3}\right)\right]$ by Lemma A.1. Finally $C\left[\operatorname{rec}\left(S^{p_{i}-m}(0), v_{2}, v_{3}\right)\right] \rightsquigarrow{ }^{p_{i}-m} C^{\prime}\left[\operatorname{rec}\left(0, v_{2}, v_{3}\right)\right]$ by Lemma A.9 and we can conclude since $p \geqslant m$ and thus $p+p_{i}-m \geqslant p_{i}$. 


\section{Appendix B Subject reduction for Loop ${ }^{\omega}$}

\section{B.1 Substitution and renaming}

Recall that renaming is only defined for mutable variables and that the imperative substitution is only defined for read-only variables. Since we consider commands up to $\alpha$-conversion, we may assume that no variable capture can occur.

Definition B.1. The renaming meta-operation on commands, sequences and expressions, denoted respectively by $c[x \hookleftarrow y],\{s\}_{z}[x \hookleftarrow y]$ and $e^{\prime}[x \hookleftarrow y]$ is defined in Table B.1.

\begin{tabular}{|c|c|c|}
\hline $\begin{array}{l}x[x \hookleftarrow y] \\
z[x \hookleftarrow y] \\
w[x \hookleftarrow y]\end{array}$ & $\begin{array}{l}=y \\
=z \\
=w\end{array}$ & if $x \neq z$ \\
\hline $\begin{array}{l}\varepsilon[x \hookleftarrow y] \\
(c ; s)[x \hookleftarrow y] \\
\left(\operatorname{cst} x=e^{\prime} ; s\right)[x \hookleftarrow y] \\
\left(\operatorname{cst} z=e^{\prime} ; s\right)[x \hookleftarrow y] \\
\left(\operatorname{var} x: \tau:=e^{\prime} ; s\right)[x \hookleftarrow y] \\
\left(\operatorname{var} z: \tau:=e^{\prime} ; s\right)[x \hookleftarrow y]\end{array}$ & $\begin{array}{l}=\varepsilon \\
=c[x \hookleftarrow y] ; s[x \hookleftarrow y] \\
=\text { cst } x=\left(e^{\prime}[x \hookleftarrow y]\right) ; s \\
=\operatorname{cst} z=\left(e^{\prime}[x \hookleftarrow y]\right) ;(s[x \hookleftarrow y]) \\
=\operatorname{var} x: \tau:=\left(e^{\prime}[x \hookleftarrow y]\right) ; s \\
=\operatorname{var} z: \tau:=\left(e^{\prime}[x \hookleftarrow y]\right) ;(s[x \hookleftarrow y])\end{array}$ & $\begin{array}{l}\text { if } x, y \neq z \\
\text { if } x, y \neq z\end{array}$ \\
\hline $\begin{array}{l}\{s\}_{\vec{z}}[x \hookleftarrow y] \\
\left(\text { for } x:=1 \text { to } e^{\prime}\{s\}_{\vec{r}}\right)[x \hookleftarrow y] \\
\left(\text { for } z:=1 \text { to } e^{\prime}\{s\}_{\vec{r}}\right)[x \hookleftarrow y] \\
\left(x:=e^{\prime}\right)[x \hookleftarrow y] \\
\left(z:=e^{\prime}\right)[x \hookleftarrow y] \\
(\operatorname{inc}(x))[x \hookleftarrow y] \\
(\operatorname{inc}(z))[x \hookleftarrow y] \\
(\operatorname{dec}(x))[x \hookleftarrow y] \\
(\operatorname{dec}(z))[x \hookleftarrow y] \\
\left(e^{\prime}\left(\vec{e}^{\prime \prime}, \vec{y}\right)\right)[x \hookleftarrow y]\end{array}$ & $\begin{array}{l}=\{s[x \hookleftarrow y]\}_{\vec{z}[x \hookleftarrow y]} \\
=\text { for } x:=1 \text { to }\left(e^{\prime}[x \hookleftarrow y]\right)\{s\}_{\vec{r}} \\
=\text { for } z:=1 \text { to }\left(e^{\prime}[x \hookleftarrow y]\right)\left(\{s\}_{\vec{r}}[x \hookleftarrow y]\right) \\
=y:=\left(e^{\prime}[x \hookleftarrow y]\right) \\
=z:=\left(e^{\prime}[x \hookleftarrow y]\right) \\
=\operatorname{inc}(y) \\
=\operatorname{inc}(z) \\
=\operatorname{dec}(y) \\
=\operatorname{dec}(z) \\
=e^{\prime}[x \hookleftarrow y]\left(\vec{e}^{\prime \prime}[x \hookleftarrow y], \vec{y}[x \hookleftarrow y]\right)\end{array}$ & $\begin{array}{l}\text { if } x, y \neq z \\
\text { if } x \neq z \\
\text { if } x \neq z \\
\text { if } x \neq z\end{array}$ \\
\hline
\end{tabular}

Table B.1. The renaming meta-operation

Definition B.2. The substitution meta-operation on commands, sequences and expressions, denoted respectively by $c[x \leftarrow e],\{s\}_{z}[x \leftarrow e]$ and $e^{\prime}[x \leftarrow e]$ is defined in Table B.2.

Lemma B.3. If $\Gamma, x: \tau ; \Omega \vdash c: \mathcal{T}$ and $\emptyset ; \emptyset \vdash e: \tau$ then $\Gamma ; \Omega \vdash c[x \leftarrow e]: \mathcal{T}$.

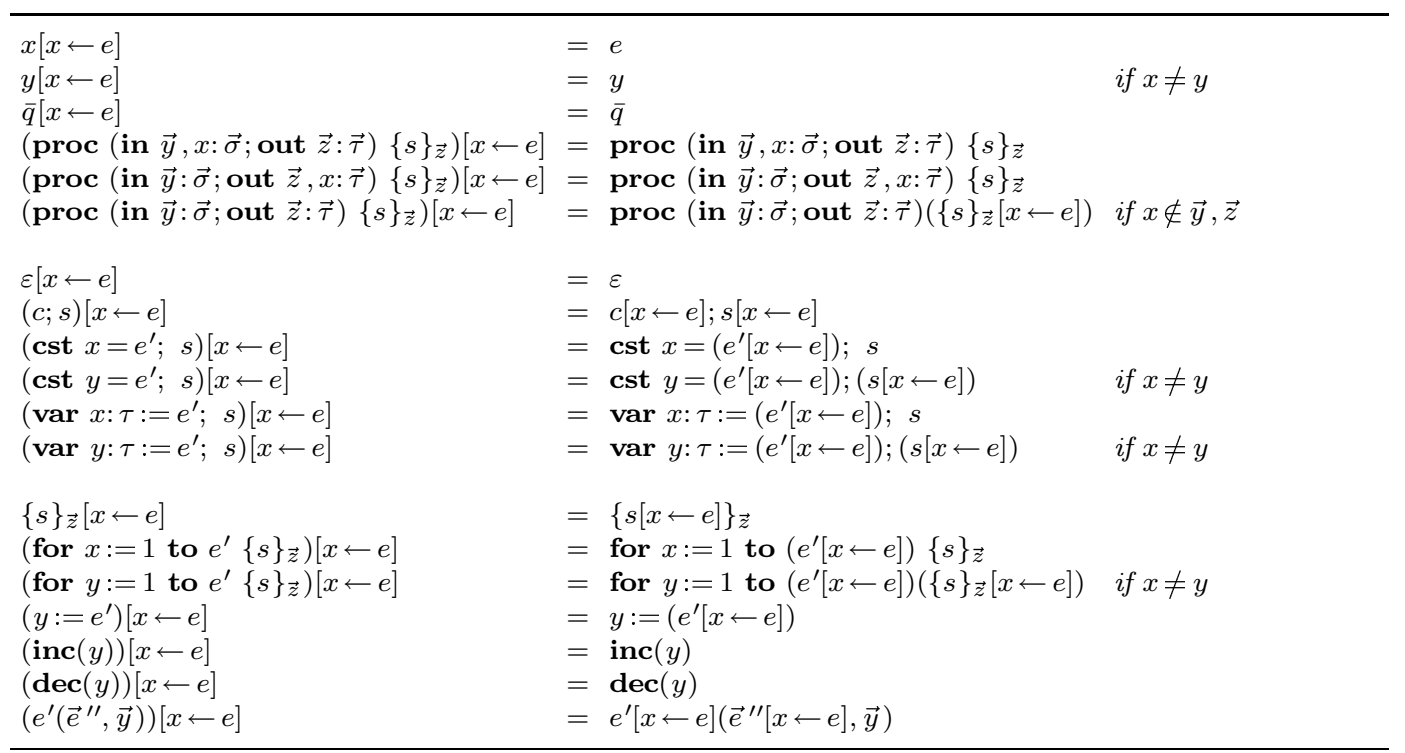

Table B.2. The substitution meta-operation 
Proof. Straightforward induction on $c$.

Lemma B.4. If $\Gamma$; $x: \tau, \Omega \vdash c: \mathcal{T}$ then $\Gamma ; y: \tau, \Omega \vdash c[x \hookleftarrow y]: \mathcal{T}$.

Proof. Straightforward induction on $c$.

\section{B.2 Preliminary lemmas}

Lemma B.5. If $\Omega \vdash \mu$ and for all $\Delta \subset \Omega, \emptyset ; \Delta \vdash e: \tau$ and $e={ }_{\mu} w$, then we have $\emptyset ; \emptyset \vdash w: \tau$.

Proof. The case $e=w$ is trivial and if $e$ is some variable $x$ then by definition of $\Omega \vdash \mu$, we have $\emptyset ; \emptyset \vdash \mu(x)=w: \tau$.

Lemma B.6. If $\Omega \vdash \mu$, then for all $\Gamma \subset \Omega$, we have $\Gamma \vdash \mu$.

Proof. By definition of store typing.

\section{B.3 Proof of Theorem 3.18}

TheOREM 3.18. For any environment $\Omega$ and any state $(c, \mu)$, we have that

- $\emptyset ; \Omega \vdash(c, \mu)$ and $(c, \mu) \mapsto\left(c^{\prime}, \mu^{\prime}\right)$ implies $\emptyset ; \Omega \vdash\left(c^{\prime}, \mu^{\prime}\right)$

- $\emptyset ; \Omega \vdash(s, \mu)$ and $(s, \mu) \mapsto\left(s^{\prime}, \mu^{\prime}\right)$ implies $\emptyset ; \Omega \vdash\left(s^{\prime}, \mu^{\prime}\right)$

and $\operatorname{dom}(\mu)=\operatorname{dom}\left(\mu^{\prime}\right)$.

Proof. By induction on the derivation of $(c, \mu) \mapsto\left(c^{\prime}, \mu^{\prime}\right)$, and then by analysis of the typing derivation.

- $\quad$ (S.BLOCK): we have $\Omega \vdash \mu$ and

$$
\frac{\vec{z} \subset \Omega \quad \Gamma ; \vec{z}: \vec{\sigma} \vdash s: \text { seq }}{\emptyset ; \Omega \vdash\{s\}_{\vec{z}}: \text { comm }}
$$

By Lemma B.6 we get $\vec{z}: \vec{\sigma} \vdash \mu$, and by induction hypothesis on $\vec{z}: \vec{\sigma} \vdash(s, \mu)$, we obtain $\operatorname{dom}(\mu)=\operatorname{dom}\left(\mu^{\prime}\right)$ and $\vec{z}: \vec{\sigma} \vdash\left(s^{\prime}, \mu^{\prime}\right)$ which gives us $\emptyset ; \Omega \vdash s^{\prime}$ : seq and $\Omega \vdash \mu^{\prime}$. We can build the following typing derivation:

$$
\frac{\vec{z} \subset \Omega \quad \Gamma ; \vec{z}: \vec{\sigma} \vdash s^{\prime}: \text { seq }}{\emptyset ; \Omega \vdash\left\{s^{\prime}\right\}_{\vec{z}}: \text { comm }}
$$

- $\quad$ (S.SEQ-I): we have $\Omega \vdash \mu$ and

$$
\frac{\frac{\vec{z} \subset \Omega \quad \overline{\emptyset ; \vec{z}: \vec{\sigma} \vdash \varepsilon: \text { seq }}}{\emptyset ; \Omega \vdash\{\}_{\vec{z}}: \text { comm }} \quad \emptyset ; \Omega \vdash s: \text { seq }}{\emptyset ; \Omega \vdash\{\}_{\vec{z}} ; s: \text { seq }}
$$

then we get $\Omega \vdash \mu$ and $\emptyset ; \Omega \vdash s$ : seq.

- $\quad$ (S.SEQ-II): we have $\Omega \vdash \mu$ and

$$
\frac{\emptyset ; \Omega \vdash c: \text { comm } \quad \emptyset ; \Omega \vdash s: \text { seq }}{\emptyset ; \Omega \vdash c ; s: \text { seq }}
$$

By induction hypothesis on $\Omega \vdash(c, \mu)$, we obtain $\operatorname{dom}(\mu)=\operatorname{dom}\left(\mu^{\prime}\right)$ and $\Omega \vdash\left(c^{\prime}, \mu^{\prime}\right)$ which gives us $\emptyset ; \Omega \vdash c^{\prime}$ : comm and $\Omega \vdash \mu^{\prime}$. We can build the following typing derivation:

$$
\frac{\emptyset ; \Omega \vdash c^{\prime}: \text { comm } \quad \emptyset ; \Omega \vdash s: \text { seq }}{\emptyset ; \Omega \vdash c^{\prime} ; s: \text { seq }}
$$


- (S.VAR-I): we have $\Omega \vdash \mu$ and

$$
\frac{\emptyset ; \Omega \vdash e: \tau \quad \emptyset ; \Omega, y: \tau \vdash s: \text { seq }}{\emptyset ; \Omega \vdash \operatorname{var} y: \tau:=e ; s: \text { seq }}
$$

By Lemma B.5, $\emptyset ; \Omega \vdash e: \tau, \Omega \vdash \mu$ and $e={ }_{\mu} w$ implies $\emptyset ; \emptyset \vdash w: \tau$. By definition of store typing, $\Omega \vdash \mu$ then implies $\Omega, y: \tau \vdash(\mu, y \leftarrow w)$. By induction hypothesis, since $\Omega, y: \tau \vdash(s,(\mu, y \leftarrow w))$ is derivable, we obtain $\operatorname{dom}(\mu, y \leftarrow w)=\operatorname{dom}\left(\mu^{\prime}, y \leftarrow w^{\prime}\right)$, that is $\operatorname{dom}(\mu)=\operatorname{dom}\left(\mu^{\prime}\right)$, and $\Omega, y: \tau \vdash\left(\varepsilon,\left(\mu^{\prime}, y \leftarrow w^{\prime}\right)\right)$ which gives us $\Omega, y: \tau \vdash \mu^{\prime}$, $y \leftarrow w^{\prime}$, that is $\Omega \vdash \mu^{\prime}$. We get $\emptyset ; \Omega \vdash \varepsilon$ : seq.

- (S.VAR-II): we have $\Omega \vdash \mu$ and

$$
\frac{\emptyset ; \Omega \vdash e: \tau \quad \emptyset ; \Omega, y: \tau \vdash s: \text { seq }}{\emptyset ; \Omega \vdash \operatorname{var} y: \tau:=e ; s: \text { seq }}
$$

By Lemma B.5, $\emptyset ; \Omega \vdash e: \tau, \Omega \vdash \mu$ and $e={ }_{\mu} w$ implies $\emptyset ; \emptyset \vdash w: \tau$. By definition of store typing, $\Omega \vdash \mu$ then implies $\Omega, y: \tau \vdash(\mu, y \leftarrow w)$. By induction hypothesis, since $\emptyset ; \Omega, y: \tau \vdash(s,(\mu, y \leftarrow w))$ is derivable, we obtain $\operatorname{dom}(\mu, y \leftarrow w)=\operatorname{dom}\left(\mu^{\prime}, y \leftarrow w^{\prime}\right)$ which implies $\operatorname{dom}(\mu)=\operatorname{dom}\left(\mu^{\prime}\right)$, and $\Omega, y: \tau \vdash\left(s^{\prime},\left(\mu, y \leftarrow w^{\prime}\right)\right)$ which implies $\emptyset$; $\Omega \vdash \operatorname{var} y: \tau:=w^{\prime} ; s^{\prime}$ and $\Omega, y: \tau \vdash\left(\mu^{\prime}, y \leftarrow w^{\prime}\right)$. This last assertion trivially implies $\Omega \vdash \mu^{\prime}$ by definition of store typing, and $\emptyset ; \emptyset \vdash w^{\prime}: \tau$. We can then build the following typing derivation to conclude:

$$
\frac{\emptyset ; \Omega \vdash w^{\prime}: \tau \quad \emptyset ; \Omega, y: \tau \vdash s^{\prime}: \mathbf{s e q}}{\emptyset ; \Omega \vdash \operatorname{var} y: \tau:=w^{\prime} ; s^{\prime}: \mathbf{s e q}}
$$

- (S.ASSIGN): we have $\Omega \vdash \mu$ and

$$
\frac{\frac{y: \tau \in \Omega \quad \emptyset ; \Omega \vdash e: \tau}{\emptyset ; \Omega \vdash y:=e: \text { comm }} \quad \emptyset ; \Omega \vdash s: \text { seq }}{\emptyset ; \Omega \vdash y:=e ; s: \text { seq }}
$$

then we get $\emptyset ; \Omega \vdash s$ : seq. By Lemma B.5, we have $\emptyset ; \emptyset \vdash w: \tau$, and since $y: \tau \in \Omega$ we get $\Omega \vdash \mu[y \leftarrow w]$ and $\operatorname{dom}(\mu)=\operatorname{dom}(\mu[y \leftarrow w])$.

- (S.INC): we have $\Omega \vdash \mu$ and

then we easily get $\Omega \vdash \mu$ and

$$
\frac{y: \operatorname{int} \in \Omega}{\emptyset ; \Omega \vdash \operatorname{inc}(y): \text { comm }}
$$

- (S.DEC): similar to above.

$$
\frac{y: \text { int } \in \Omega \quad \emptyset ; \Omega \vdash \overline{q+1}: \text { int }}{\emptyset ; \Omega \vdash y:=\overline{q+1} \text { comm }}
$$

- (S.CALL): we have $\Omega \vdash \mu$ and

$$
\frac{\emptyset ; \Omega \vdash p: \operatorname{proc}(\text { in } \vec{\sigma} ; \text { out } \vec{\tau}) \quad \emptyset ; \Omega \vdash e_{i}: \sigma_{i} \quad r_{j}: \tau_{j} \in \Omega}{\emptyset ; \Omega \vdash p(\vec{e}, \vec{r}): \text { comm }}
$$

By Lemma B.5, $\emptyset ; \Omega \vdash e_{i}: \sigma_{i}, \Omega \vdash \mu$ and $\vec{e}={ }_{\mu} \vec{w}$ implies $\emptyset ; \emptyset \vdash w_{i}$ : $\sigma_{i}$. Still by Lemma B.5, $\emptyset ; \Omega \vdash p$ : $\operatorname{proc}($ in $\vec{\sigma} ;$ out $\vec{\tau}), \Omega \vdash \mu$ and $p={ }_{\mu} \operatorname{proc}($ in $\vec{y}: \vec{\sigma}$; out $\vec{z}: \vec{\tau})\{s\}_{\vec{z}}$ implies $\emptyset ; \emptyset \vdash \operatorname{proc}($ in $\vec{y}: \vec{\sigma} ;$ out $\vec{z}: \vec{\tau})\{s\}_{\vec{z}}$ : $\operatorname{proc}($ in $\vec{\sigma} ;$ out $\vec{\tau})$, that is

$$
\frac{\vec{z} \neq \emptyset \quad \emptyset ; \vec{y}: \vec{\sigma} ; \vec{z}: \vec{\tau} \vdash s: \text { seq }}{\emptyset ; \emptyset \vdash \text { proc (in } \vec{y}: \vec{\sigma} ; \text { out } \vec{z}: \vec{\tau})\{s\}_{\vec{z}}: \operatorname{proc}(\text { in } \vec{\sigma} ; \text { out } \vec{\tau})}
$$

By Lemmas B.3 and B.4, $\vec{y}: \vec{\sigma} ; \vec{z}: \vec{\tau} \vdash s$ : comm and $\emptyset ; \emptyset \vdash w_{i}: \sigma_{i}$ implies $\emptyset ; \vec{r}$ : $\vec{\tau} \vdash s[\vec{y} \leftarrow \vec{w}][\vec{z} \hookleftarrow \vec{r}]$ : comm, and since $r_{j}: \tau_{j} \in \Omega$, the typing rule (т.сомM) gives us $\emptyset ; \Omega \vdash\{s[\vec{y} \leftarrow \vec{w}][\vec{z} \hookleftarrow \vec{r}]\}_{\vec{r}}$ : comm. By Lemma 3.11, we have $\emptyset ; \emptyset \vdash \epsilon\left(\tau_{j}\right): \tau_{j}$, and since $r_{j}: \tau_{j} \in \Omega$, we obtain $\Omega \vdash \mu[\vec{r} \leftarrow \epsilon(\vec{\tau})]$. 
- (S.CST): we have $\Omega \vdash \mu$ and

$$
\frac{\emptyset ; \Omega \vdash e: \tau \quad y: \tau ; \Omega \vdash s: \mathbf{s e q}}{\emptyset ; \Omega \vdash \operatorname{cst} y=e ; s: \text { seq }}
$$

We trivially have $\Omega \vdash \mu$. By Lemma B.5, $\emptyset ; \Omega \vdash e: \tau, \Omega \vdash \mu$ and $e={ }_{\mu} w$ implies $\emptyset$; $\emptyset \vdash w: \tau$. By Lemma B.3, $y: \tau ; \Omega \vdash s$ : seq and $\emptyset ; \emptyset \vdash w: \tau$ implies $\emptyset ; \Omega \vdash s[y \leftarrow w]$ : seq.

- $\quad$ (S.FOR-I): we have $\Omega \vdash \mu$ and

$$
\frac{\vec{z} \subset \Omega \quad \emptyset ; \Omega \vdash e: \text { int } \quad y: \text { int } ; \vec{z}: \vec{\sigma} \vdash s: \text { seq }}{\emptyset ; \Omega \vdash \text { for } y:=1 \text { to } e\{s\}_{\vec{z}}: \text { comm }}
$$

We clearly have $\Omega \vdash \mu$ and $\emptyset ; \Omega \vdash\{\}_{\vec{z}}$ : comm.

- $\quad$ (S.FOR-II): we have $\Omega \vdash \mu$ and

$$
\frac{\vec{z} \subset \Omega \quad \emptyset ; \Omega \vdash e: \text { int } \quad y: \text { int } ; \vec{z}: \vec{\sigma} \vdash s: \text { seq }}{\emptyset ; \Omega \vdash \text { for } y:=1 \text { to } e\{s\}_{\vec{z}}: \text { comm }}
$$

\begin{tabular}{|c|c|c|}
\hline & $\vec{z} \subset \vec{z}: \vec{\sigma} \quad \emptyset ; \Omega \vdash \bar{q}:$ int $\quad y:$ int $; \vec{z}: \vec{\sigma} \vdash s: \mathbf{s e q}$ & \\
\hline & $\emptyset ; \vec{z}: \vec{\sigma} \vdash$ for $y:=1$ to $\bar{q}\{s\}_{\vec{z}}:$ comm & $\emptyset ; \vec{z}: \vec{\sigma} \vdash s[y \leftarrow \overline{q+1}]:$ seq \\
\hline$\vec{z} \subset \Omega$ & $\emptyset ; \vec{z}: \vec{\sigma} \vdash$ for $y:=1$ to $\bar{q}\{s\}_{\vec{z}}$ & $\leftarrow \overline{q+1}]:$ seq \\
\hline
\end{tabular}

By Lemmas B.3, y: int; $\vec{z}: \vec{\tau} \vdash s$ : seq and $\emptyset ; \emptyset \vdash \overline{q+1}$ : int implies $\emptyset ; \vec{z}: \vec{\tau} \vdash s[y \leftarrow$ $\overline{q+1}$ : seq. We clearly have $\Omega \vdash \mu$ and the following rule is easily derivable:

\section{Appendix C Translation ( ${ }^{\star}$ is type-preserving}

\section{C.1 Preliminary lemmas}

Lemma C.1. For any environment $\Gamma$, variable $y$, and terms $t$ and e of System $T$, if $\Gamma$, $y: \tau \vdash t: \tau^{\prime}$ and $\Gamma, \Omega \vdash e: \tau$ then $\Gamma, \Omega \vdash t[e / y]: \tau^{\prime}$.

Proof. By induction on $t$.

Lemma C.2. For any environment $\Gamma$, variable $y$, type $\tau^{\prime}$ and term $t$ of System $T$, if $y \notin F I d(t)$ then $\Gamma, y: \tau^{\prime} \vdash t: \tau$ implies $\Gamma \vdash t: \tau$.

Proof. By induction on the typing judgment of $t$.

Notation C.3. For any environment $\Gamma=\left(x_{1}: \tau_{1}, \ldots, x_{n}: \tau_{n}\right)$, we write $\Gamma^{\times}$for $\tau_{1} \times \ldots \times \tau_{n}$.

Lemma C.4. For any environment $\Gamma$, $\operatorname{dom}(\Gamma)=\operatorname{dom}\left(\Gamma^{\star}\right)$ and $x: \tau \in \Gamma$ implies $x: \tau^{\star} \in \Gamma^{\star}$.

\section{C.2 Proof of Theorem 4.7}

TheOrem 4.7. For any environments $\Gamma$ and $\Omega$, any expression $e$ and any sequence $s$ and any block $\{s\}_{\vec{x}}$ we have:

- $\quad \Gamma ; \Omega \vdash e: \tau$ implies $\Gamma^{\star}, \Omega^{\star} \vdash e^{\star}: \tau^{\star}$

- $\quad \Gamma ; \Omega, \vec{x}: \vec{\sigma} \vdash s$ : seq implies $\Gamma^{\star}, \Omega^{\star}, \vec{x}: \vec{\sigma}^{\star} \vdash(s)_{\vec{x}}^{\star}:\left(\vec{\sigma}^{\star}\right)^{\times}$

- $\quad \Gamma ; \Omega, \vec{x}: \vec{\sigma} \vdash\{s\}_{\vec{x}}: \mathbf{c o m m}$ implies $\Gamma^{\star}, \Omega^{\star}, \vec{x}: \vec{\sigma}^{\star} \vdash\{s\}_{\vec{x}}^{\star}: \vec{\sigma}^{\times}$ 
Proof. By induction on $e, s$, and $\{s\}_{\vec{x}}$.

- $e=\bar{n}$. We have:

$$
\Gamma ; \Omega \vdash \bar{n}: i n t
$$

and then

$$
\Gamma^{\star}, \Omega^{\star} \vdash S^{n}(0): \text { int }
$$

is clearly derivable.

- $e=y$. We have:

and then

$$
\frac{y: \tau \in \Gamma, \Omega}{\Gamma ; \Omega \vdash y: \tau}
$$

$$
\Gamma^{\star}, \Omega^{\star} \vdash y: \tau^{\star}
$$

since by Lemma C.4, $y: \tau \in \Gamma, \Omega$ implies $y: \tau^{\star} \in \Gamma^{\star}, \Omega^{\star}$.

- $e=\operatorname{proc}($ in $\vec{y}: \vec{\sigma} ;$ out $\vec{z}: \vec{\tau})\{s\}_{\vec{z}}$. We have:

$$
\frac{\vec{z} \neq \emptyset \quad \Gamma, \vec{y}: \vec{\sigma} ; \vec{z}: \vec{\tau} \vdash s: \text { seq }}{\Gamma ; \Omega \vdash \operatorname{proc}(\vec{y}: \text { in } \vec{\sigma} ; \vec{z}: \text { out } \vec{\tau})\{s\}_{\vec{z}}: \text { proc }(\text { in } \vec{\sigma} ; \text { out } \vec{\tau})}
$$

Since $\Gamma, \vec{y}: \vec{\sigma} ; \vec{z}: \vec{\tau} \vdash\{s\}_{\vec{z}}:$ comm is derivable, we obtain by induction hypothesis $\Gamma^{\star}$, $\vec{y}: \vec{\sigma}^{\star}, \vec{z}: \vec{\tau}^{\star} \vdash\{s\}_{\vec{z}}^{\star}:\left(\vec{\tau}^{\star}\right)^{\star}$, and by Lemma $4.2 \vdash \delta\left(\tau_{i}\right): \tau_{i}^{\star}$. By Lemma C.1, we then get $\Gamma^{\star}, \vec{y}: \vec{\sigma}^{\star}, \Omega^{\star} \vdash\{s\}_{\vec{z}}^{\star}[\delta(\vec{\tau}) / \vec{z}]:\left(\vec{\tau}^{\star}\right)^{\times}$. We build the following typing derivation:

$$
\frac{\Gamma^{\star}, \vec{y}: \vec{\sigma}^{\star}, \Omega^{\star} \vdash\{s\}_{z}^{\star}[\delta(\vec{\tau}) / \vec{z}]:\left(\vec{\tau}^{\star}\right)^{\times}}{\Gamma^{\star}, \Omega^{\star} \vdash \mathbf{f n}\left(\vec{y}: \vec{\sigma}^{\star}\right) \Rightarrow\{s\}_{z}^{\star}[\delta(\vec{\tau}) / \vec{z}]:\left(\vec{\sigma}^{\star}\right)^{\times} \rightarrow\left(\vec{\tau}^{\star}\right)^{\times}}
$$

- $\{s\}_{\vec{x}}$. We have:

$$
\frac{\vec{x} \subset \Omega, \vec{x}: \vec{\sigma} \quad \Gamma ; \vec{x}: \vec{\sigma} \vdash s: \text { seq }}{\Gamma ; \Omega, \vec{x}: \vec{\sigma} \vdash\{s\}_{\vec{x}}: \text { comm }}
$$

Since $\Gamma ; \vec{x}: \vec{\sigma} \vdash s$ : seq is derivable, we obtain by induction hypothesis the required typing derivation $\Gamma^{\star} ; \vec{x}: \vec{\sigma}^{\star} \vdash(s)_{\vec{x}}^{\star}:\left(\vec{\sigma}^{\star}\right)^{\times}$.

- $s=\varepsilon$. We have:

$$
\overline{\Gamma ; \Omega, \vec{x}: \vec{\sigma} \vdash \varepsilon: \mathbf{s e q}}
$$

and then $\Gamma^{\star}, \Omega^{\star}, \vec{x}: \vec{\sigma}^{\star} \vdash \vec{x}:\left(\vec{\sigma}^{\star}\right)^{\times}$by Lemma C.4.

- $s=\operatorname{var} y: \tau:=e ; s^{\prime}$ with $s^{\prime} \neq \varepsilon$. We have:

$$
\frac{\Gamma ; \Omega, \vec{x}: \vec{\sigma} \vdash e: \tau \quad \Gamma ; \Omega, \vec{x}: \vec{\sigma}, y: \tau \vdash s^{\prime}: \text { seq }}{\Gamma ; \Omega, \vec{x}: \vec{\sigma} \vdash \operatorname{var} y: \tau:=e ; s^{\prime}: \text { seq }}
$$

By induction hypothesis, we obtain $\Gamma^{\star}, \Omega^{\star}, \vec{x}: \vec{\sigma}^{\star}, y: \tau^{\star} \vdash\left(s^{\prime}\right){ }_{\vec{x}}^{\star}:\left(\vec{\sigma}^{\star}\right)^{\star}$ and $\Gamma^{\star}, \Omega^{\star}, \vec{x}$ :

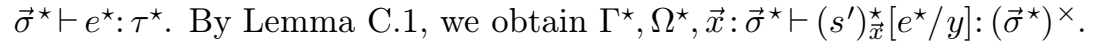

- $s=\mathbf{c s t} y=e ; s^{\prime}$. We have:

$$
\frac{\Gamma ; \Omega, \vec{x}: \vec{\sigma} \vdash e: \tau \quad \Gamma, y: \tau ; \Omega, \vec{x}: \vec{\sigma} \vdash s^{\prime}: \text { seq }}{\Gamma ; \Omega, \vec{x}: \vec{\sigma} \vdash \text { cst } y=e ; s^{\prime}: \text { seq }}
$$

By induction hypothesis, we obtain $\Gamma^{\star}, \Omega^{\star}, \vec{x}: \vec{\sigma}^{\star}, y: \tau^{\star} \vdash\left(s^{\prime}\right){ }_{\vec{x}}^{\star}:\left(\vec{\sigma}^{\star}\right)^{\star}$ and $\Gamma^{\star}, \Omega^{\star}, \vec{x}$ : $\vec{\sigma}^{\star} \vdash e^{\star}: \tau^{\star}$. We build then the following typing derivation :

$$
\frac{\Gamma^{\star}, \Omega^{\star}, \vec{x}: \vec{\sigma}^{\star} \vdash e^{\star}: \tau^{\star} \quad \Gamma^{\star}, \Omega^{\star}, \vec{x}: \vec{\sigma}^{\star}, y: \tau^{\star} \vdash\left(s^{\prime}\right)_{\vec{x}}^{\star}:\left(\vec{\sigma}^{\star}\right)^{\times}}{\Gamma^{\star}, \Omega^{\star}, \vec{x}: \vec{\sigma}^{\star} \vdash \text { let } y=e^{\star} \text { in }\left(s^{\prime}\right)_{\vec{x}}^{\star}:\left(\vec{\sigma}^{\star}\right)^{\star}}
$$


- $s=y:=e ; s^{\prime}$. We have:

$$
\frac{\frac{y: \tau \in \Omega, \vec{x}: \vec{\sigma} \quad \Gamma ; \Omega, \vec{x}: \vec{\sigma} \vdash e: \tau}{\Gamma ; \Omega, \vec{x}: \vec{\sigma} \vdash y:=e: \mathbf{c o m m}} \quad \Gamma ; \Omega, \vec{x}: \vec{\sigma} \vdash s^{\prime}: \mathbf{s e q}}{\Gamma ; \Omega, \vec{x}: \vec{\sigma} \vdash y:=e, s^{\prime}: \text { seq }}
$$

By induction hypothesis on $\Gamma ; \Omega, \vec{x}: \vec{\sigma} \vdash e: \tau$, we obtain $\Gamma^{\star}, \Omega^{\star}, \vec{x}: \vec{\sigma}^{\star} \vdash e^{\star}: \tau^{\star}$ and by induction hypothesis on $\Gamma ; \Omega, \vec{x}: \vec{\sigma} \vdash s^{\prime}$ : seq, we obtain $\Gamma^{\star}, \Omega^{\star}, \vec{x}: \vec{\sigma}^{\star} \vdash\left(s^{\prime}\right)_{\vec{x}}^{\star}:\left(\vec{\sigma}^{\star}\right)^{\times}$. We build then the following typing derivation :

$$
\frac{\Gamma^{\star}, \Omega^{\star}, \vec{x}: \vec{\sigma}^{\star} \vdash e^{\star}: \tau^{\star} \quad \Gamma^{\star}, \Omega^{\star}, \vec{x}: \vec{\sigma}^{\star} \vdash\left(s^{\prime}\right)_{\vec{x}}^{\star}:\left(\vec{\sigma}^{\star}\right)^{\star}}{\Gamma^{\star}, \Omega^{\star}, \vec{x}: \vec{\sigma}^{\star} \vdash \text { let } y=e^{\star} \operatorname{in}\left(s^{\prime}\right)_{\vec{x}}^{\star}:\left(\vec{\sigma}^{\star}\right)^{\star}}
$$

- $s=\operatorname{inc}(y) ; s^{\prime}$. We have:

$$
\begin{gathered}
\frac{y: \operatorname{int} \in \Omega, \vec{x}: \vec{\sigma}}{\Gamma ; \Omega, \vec{x}: \vec{\sigma} \vdash \operatorname{inc}(y): \operatorname{comm}} \quad \Gamma ; \Omega, \vec{x}: \vec{\sigma} \vdash s^{\prime}: \mathbf{s e q} \\
\Gamma ; \Omega, \vec{x}: \vec{\sigma} \vdash \operatorname{inc}(y) ; s^{\prime}: \mathbf{s e q}
\end{gathered}
$$

We remark that since $y:$ int $\in \Omega, \vec{x}: \vec{\sigma}$, by Lemma C.4, we get (y: int) $\in \Omega^{\star}, \vec{x}: \vec{\sigma}^{\star}$ and we have $\Omega^{\star}, \vec{x}: \vec{\sigma}^{\star}, y:$ int $=\Omega^{\star}, \vec{x}: \vec{\sigma}^{\star}$. By induction hypothesis, we obtain $\Gamma^{\star}, \Omega^{\star}$, $\vec{x}: \vec{\sigma}^{\star} \vdash\left(s^{\prime}\right)_{\vec{x}}^{\star}:\left(\vec{\sigma}^{\star}\right)^{\star}$. We can then build the following typing derivation :

$$
\frac{\frac{\Gamma^{\star}, \Omega^{\star}, \vec{x}: \vec{\sigma}^{\star} \vdash y: \text { int }}{\Gamma^{\star}, \Omega^{\star}, \vec{x}: \vec{\sigma}^{\star} \vdash \operatorname{succ}(y): \text { int }} \quad \Gamma^{\star}, \Omega^{\star}, \vec{x}: \vec{\sigma}^{\star} \vdash\left(s^{\prime}\right)_{\vec{x}}^{\star}:\left(\vec{\sigma}^{\star}\right)^{\times}}{\Gamma^{\star}, \Omega^{\star}, \vec{x}: \vec{\sigma}^{\star} \vdash \text { let } y=\operatorname{succ}(y) \text { in }\left(s^{\prime}\right)_{\vec{x}}^{\star}:\left(\vec{\sigma}^{\star}\right)^{\star}}
$$

- $s=\operatorname{dec}(y) ; s^{\prime}$. Similar to the previous case.

- $s=p(\vec{e} ; \vec{z}) ; s^{\prime}$. We have:

$\frac{\frac{\Gamma ; \Omega, \vec{x}: \vec{\sigma} \vdash p: \text { proc }\left(\text { in } \vec{\tau} ; \text { out } \vec{\tau}^{\prime}\right) \quad \Gamma ; \Omega, \vec{x}: \vec{\sigma} \vdash e_{i}: \tau_{i} \quad z_{j}: \tau_{j}^{\prime} \in \Omega, \vec{x}: \vec{\sigma}}{\Gamma ; \Omega, \vec{x}: \vec{\sigma} \vdash p(\vec{e} ; \vec{z}): \mathbf{c o m m}} \quad \Gamma ; \Omega, \vec{x}: \vec{\sigma} \vdash s^{\prime}: \text { seq }}{\Gamma ; \Omega, \vec{x}: \vec{\sigma} \vdash p(\vec{e} ; \vec{z}) ; s^{\prime}: \text { seq }}$

By induction hypothesis, we obtain $\Gamma^{\star}, \Omega^{\star}, \vec{x}: \vec{\sigma}^{\star} \vdash e_{i}^{\star}: \tau_{i}^{\star}$ and $\Gamma^{\star}, \Omega^{\star}, \vec{x}: \vec{\sigma}^{\star} \vdash p^{\star}$ : $\left(\vec{\tau}^{\star}\right)^{\times} \rightarrow\left(\vec{\tau}^{\prime \star}\right)^{\times}$and $\Gamma^{\star}, \Omega^{\star}, \vec{x}: \vec{\sigma}^{\star} \vdash\left(s^{\prime}\right)_{\vec{x}}^{\star}:\left(\vec{\sigma}^{\star}\right)^{\times}$. Note that since $z_{j}: \tau_{j}^{\prime} \in \Omega, \vec{x}: \vec{\sigma}^{\star}$, by Lemma C.4, we get $\vec{z}: \vec{\tau}^{\prime \star} \subset \Omega^{\star}, \vec{x}: \vec{\sigma}^{\star}$ and then $\Omega^{\star}, \vec{x}: \vec{\sigma}^{\star}, \vec{z}:\left(\vec{\tau}^{\prime \star}\right)^{\star}=\Omega^{\star}, \vec{x}: \vec{\sigma}^{\star}$. We can then build the following typing derivation :

$$
\frac{\frac{\Gamma^{\star}, \Omega^{\star}, \vec{x}: \vec{\sigma}^{\star} \vdash p^{\star}:\left(\vec{\tau}^{\star}\right)^{\star} \rightarrow\left(\vec{\tau}^{\prime \star}\right)^{\times} \quad \Gamma^{\star}, \Omega^{\star}, \vec{x}: \vec{\sigma}^{\star} \vdash \vec{e}^{\star}: \vec{\tau}^{\star}}{\Gamma^{\star}, \Omega^{\star}, \vec{x}: \vec{\sigma}^{\star} \vdash p^{\star} \vec{e}^{\star}:\left(\vec{\tau}^{\prime \star}\right)^{\times}} \quad \Gamma^{\star}, \Omega^{\star}, \vec{x}: \vec{\sigma}^{\star} \vdash\left(s^{\prime}\right)_{\vec{x}}^{\star}:\left(\vec{\sigma}^{\star}\right)^{\times}}{\Gamma^{\star}, \Omega^{\star}, \vec{x}: \vec{\sigma}^{\star} \vdash \text { let } \vec{z}=p^{\star} \vec{e}^{\star} \text { in }\left(s^{\prime}\right)_{\vec{x}}^{\star}:\left(\vec{\sigma}^{\star}\right)^{\star}}
$$

- $s=\left\{s^{\prime}\right\}_{z}^{\star} ; s^{\prime \prime}$. We have:

$$
\frac{\Gamma ; \Omega, \vec{x}: \vec{\sigma} \vdash\left\{s^{\prime}\right\}_{\vec{z}}: \mathbf{c o m m} \quad \Gamma ; \Omega, \vec{x}: \vec{\sigma} \vdash s^{\prime \prime}: \text { seq }}{\Gamma ; \Omega, \vec{x}: \vec{\sigma} \vdash\left\{s^{\prime}\right\}_{\vec{z}}^{\star} ; s^{\prime \prime}: \text { seq }}
$$

By induction hypothesis, we obtain $\Gamma^{\star}, \Omega^{\star}, \vec{x}: \vec{\sigma}^{\star} \vdash\left\{s^{\prime}\right\}_{z}^{\star}:\left(\vec{\tau}^{\star}\right)^{\star}$ where $\vec{z}: \vec{\tau} \subset \Omega, \vec{x}: \vec{\sigma}$ (and then $\left.\Omega^{\star}, \vec{x}: \vec{\sigma}^{\star}, \vec{z}:\left(\vec{\tau}^{\star}\right)^{\times}=\Omega^{\star}, \vec{x}: \vec{\sigma}^{\star}\right)$ and $\Gamma^{\star}, \Omega^{\star}, \vec{x}: \vec{\sigma}^{\star} \vdash\left(s^{\prime \prime}\right)_{\vec{x}}^{\star}:\left(\vec{\sigma}^{\star}\right)^{\times}$. We can then build the following typing derivation :

$$
\frac{\Gamma^{\star}, \Omega^{\star}, \vec{x}: \vec{\sigma}^{\star} \vdash\left\{s^{\prime}\right\}_{\vec{z}}^{\star}:\left(\vec{\tau}^{\star}\right)^{\times} \quad \Gamma^{\star}, \Omega^{\star}, \vec{x}: \vec{\sigma}^{\star} \vdash\left(s^{\prime \prime}\right)_{\vec{x}}^{\star}:\left(\vec{\sigma}^{\star}\right)^{\times}}{\Gamma^{\star}, \Omega^{\star}, \vec{x}: \vec{\sigma}^{\star} \vdash \text { let } \vec{z}=\left\{s^{\prime}\right\}_{\vec{z}}^{\star} \text { in }\left(s^{\prime \prime}\right)_{\vec{x}}^{\star}:\left(\vec{\sigma}^{\star}\right)^{\star}}
$$

- $s=$ for $y:=1$ to $e\left\{s^{\prime}\right\}_{\vec{z}} ; s^{\prime \prime}$. We have:

$$
\frac{\frac{\vec{z} \subset \Omega, \vec{x}: \vec{\sigma} \quad \Gamma ; \Omega, \vec{x}: \vec{\sigma} \vdash e: \tau \quad \Gamma, y: \tau ; \vec{z}: \vec{\tau}^{\prime} \vdash s^{\prime}: \mathbf{s e q}}{\Gamma ; \Omega, \vec{x}: \vec{\sigma} \vdash \text { for } y:=1 \text { to } e\left\{s^{\prime}\right\} \vec{z}: \mathbf{c o m m}} \Gamma ; \Omega, \vec{x}: \vec{\sigma} \vdash s^{\prime \prime}: \text { seq }}{\Gamma ; \Omega, \vec{x}: \vec{\sigma} \vdash \text { for } y:=1 \text { to } e\left\{s^{\prime}\right\}_{\vec{z}} ; s^{\prime \prime}: \text { seq }}
$$


where $\vec{z}: \vec{\tau}^{\prime} \subset \Omega, \vec{x}: \vec{\sigma}$ (and then $\Omega^{\star}, \vec{x}: \vec{\sigma}^{\star}, \vec{z}: \vec{\tau}^{\prime \star}=\Omega^{\star}, \vec{x}: \vec{\sigma}^{\star}$ ). By induction hypothesis, we obtain $\Gamma^{\star}, y: \tau^{\star}, \vec{z}: \vec{\tau}^{\prime \star} \vdash\left\{s^{\prime}\right\}_{\vec{z}}^{\star}:\left(\vec{\tau}^{\prime \star}\right)^{\star}$ and $\Gamma^{\star}, \Omega^{\star}, \vec{x}: \vec{\sigma}^{\star} \vdash e^{\star}: \tau^{\star}$ and $\Gamma^{\star}, \Omega^{\star}, \vec{x}$ : $\vec{\sigma}^{\star} \vdash\left(s^{\prime \prime}\right)_{\vec{x}}^{\star}:\left(\vec{\sigma}^{\star}\right)^{\star}$. We build then the following typing derivation :

$$
\frac{\Gamma^{\star}, \Omega^{\star}, \vec{x}: \vec{\sigma}^{\star} \vdash e^{\star}: \tau^{\star} \quad \Gamma^{\star}, \Omega^{\star}, \vec{x}: \vec{\sigma}^{\star} \vdash \vec{z}:\left(\vec{\tau}^{\prime \star}\right)^{\times} \quad \Gamma^{\star}, \Omega^{\star}, \vec{x}: \vec{\sigma}^{\star}, y: \tau^{\star} \vdash\{s\}_{\vec{z}}^{\star}:\left(\vec{\tau}^{\prime \star}\right)^{\times}}{\Gamma^{\star}, \Omega^{\star}, \vec{x}: \vec{\sigma}^{\star} \vdash \operatorname{rec}\left(e^{\star}, \vec{z}, \lambda y \cdot \lambda \vec{z} \cdot\left\{s^{\prime}\right\}_{\vec{z}}^{\star}\right):\left(\vec{\tau}^{\prime \star}\right)^{\times}}
$$

and then:

$$
\frac{\Gamma^{\star}, \Omega^{\star}, \vec{x}: \vec{\sigma}^{\star} \vdash \operatorname{rec}\left(e^{\star}, \vec{z}, \lambda y \cdot \lambda \vec{z} \cdot\left\{s^{\prime}\right\}_{\vec{z}}^{\star}\right):\left(\vec{\tau}^{\prime \star}\right)^{\times} \quad \Gamma^{\star}, \Omega^{\star}, \vec{x}: \vec{\sigma}^{\star} \vdash\left(s^{\prime \prime}\right)_{\vec{x}}^{\star}:\left(\vec{\sigma}^{\star}\right)^{\times}}{\Gamma^{\star}, \Omega^{\star}, \vec{x}: \vec{\sigma}^{\star} \vdash \operatorname{let} \vec{z}=\operatorname{rec}\left(e^{\star}, \vec{z}, \lambda y \cdot \lambda \vec{z} \cdot\left\{s^{\prime}\right\}_{\vec{z}}^{\star}\right) \text { in }\left(s^{\prime \prime}\right)_{\vec{x}}^{\star}:\left(\vec{\sigma}^{\star}\right)^{\times}}
$$

\section{Acknowledgments}

We are grateful to Olivier Danvy for many fruitful discussions on the topics presented in this article and to Neil Jones for his early support. We also would like to thank the anonymous referees for their helpful comments.

\section{Bibliography}

[Appel, 1998] Appel, A. W. (1998). Modern Compiler Implementation in ML. Cambridge University Press, Cambridge, UK.

[Avigad and Feferman, 1998] Avigad, J. and Feferman, S. (1998). Gödel's functional ("dialectica") interpretation. In Buss, S. R., editor, Handbook of Proof Theory, pages 337-405. Elsevier Science Publishers, Amsterdam.

[Beckmann and Weiermann, 2000] Beckmann, A. and Weiermann, A. (2000). Characterizing the elementary recursive functions by a fragment of Gödel's T. Archive for Mathematical Logic, V39:475-491.

[Calude, 1988] Calude, C. (1988). Theories of computational complexity. Elsevier Science Inc., New York, NY, USA.

[Colson, 1991] Colson, L. (1991). About primitive recursive algorithms. Theoretical Computer Science, 83:57-69.

[Colson and Fredholm, 1998] Colson, L. and Fredholm, D. (1998). System T, call-by-value and the minimum problem. Theor. Comput. Sci., 206(1-2):301-315.

[Crolard et al., 2006] Crolard, T., Lacas, S., and Valarcher, P. (2006). On the Expressive Power of the Loop Language. Nordic Journal of Computing, 13(1-2):46-57.

[Davis and Weyuker, 1983] Davis, M. and Weyuker, E. (1983). Computability, Complexity and Languages. Academic Press.

[DOD, 1980] DOD (1980). The Programming Language Ada. Reference Manual. Springer, Berlin.

[Donahue, 1977] Donahue, J. E. (1977). Locations considered unnecessary. Acta Inf., 8:221-242.

[Felleisen and Friedman, 1987] Felleisen, M. and Friedman, D. P. (1987). A calculus for assignments in higher-order languages. In POPL '87: Proceedings of the 14th ACM SIGACT-SIGPLAN symposium on Principles of programming languages, page 314, New York, NY, USA. ACM Press.

[Filinski, 1994] Filinski, A. (1994). Representing monads. In Conference Record of the TwentyFirst Annual Symposium on Principles of Programming Languages, pages 446-457, Portland, Oregon.

[Filliâtre, 2003] Filliâtre, J.-C. (2003). Verification of non-functional programs using interpretations in type theory. J. Funct. Program, 13(4):709-745.

[Filliâtre and Marché, 2004] Filliâtre, J.-C. and Marché, C. (2004). Multi-prover verification of C programs. In Formal Methods and Software Engineering, 6th International Conference on Formal Engineering Methods, ICFEM 2004, volume 3308 of Lecture Notes in Computer Science, pages 15-29. Springer-Verlag.

[Gellerich and Plödereder, 2001] Gellerich, W. and Plödereder, E. (2001). Parameter-induced aliasing in ada. In Craeynest, D. and Strohmeier, A., editors, Reliable Software Technologies: Ada Europe 2001, 6th Ade-Europe International Conference Leuven, Belgium, May 14-18, 2001, Proceedings, volume 2043 of Lecture Notes in Computer Science, pages 88-99. Springer. 
[Gifford and Lucassen, 1986] Gifford, D. and Lucassen, J. (1986). Integrating functional and imperative programming. In ACM Symposium on Principles of Programming Languages.

[Girard et al., 1989] Girard, J.-Y., Lafont, Y., and Taylor, P. (1989). Proofs and Types, volume 7. Cambridge Tracts in Theorical Comp. Sci.

[Gödel, 1958] Gödel, K. (1958). Über eine bisher noch nicht benützteerweiterung des finiten standpunktes. Dialectica, 12:280-287.

[Gödel, 1990] Gödel, K. (1990). Collected Works, Volume 2. Oxford University Press, Oxford.

[Hatcliff and Danvy, 1997] Hatcliff, J. and Danvy, O. (1997). Thunks and the lambda-calculus. J. Funct. Program., 7(3):303-319.

[ISO, 2003] ISO (2003). C\# language specification ISO/IEC 23270.

[Jones, 1997] Jones, N. D. (1997). Computability and Complexity From a Programming Perspective. The MIT Press.

[Kahn, 1987] Kahn, G. (1987). Natural semantics. In Proc. Symposium on Theoretical Aspects of Computer Science (STACS), volume 247 of $L N C S$, pages 22-39. Springer-Verlag.

[Kelsey et al., 1998] Kelsey, R., Clinger, W., and Rees, J. (1998). Revised ${ }^{5}$ report on the algorithmic language Scheme. Higher-Order and Symbolic Computation, 11(1):7-105. Also appears in ACM SIGPLAN Notices 33(9), September 1998.

[Kreisel, 1951] Kreisel, G. (1951). On the interpretation of non-finitist proofs - part I. J. Symb. Log, 16(4):241-267.

[Landin, 1964] Landin, P. J. (1964). The mechanical evaluation of expressions. Computer Journal, 6:308-320.

[McCarthy, 1960] McCarthy, J. (1960). Recursive functions of symbolic expressions and their computation by machine, part I. Communications of the ACM, 3(4):184-195.

[Meyer and Ritchie, 1976] Meyer, A. R. and Ritchie, D. M. (1976). The complexity of loop programs. In Proc. ACM Nat. Meeting.

[Milner et al., 1997] Milner, R., Tofte, M., Harper, R., and MacQueen, D. (1997). The Definition of Standard ML, Revised edition. MIT Press.

[Moggi, 1991] Moggi, E. (1991). Notions of computation and monads. Information and Computation, 93(1):55-92.

[O'Hearn and Tennent, 1997] O'Hearn, P. W. and Tennent, R. D., editors (1997). Algol-like Languages. Birkhaüser.

[Peter, 1968] Peter, R. (1968). Recursive Functions. Academic Press.

[Plotkin, 1981] Plotkin, G. (1981). A structural approach to operational semantics. Technical Report DAIMI FN-19, Aarhus University.

[Reynolds, 1978] Reynolds, J. C. (1978). Syntactic control of interference. In POPL, pages 39-46.

[Reynolds, 1981] Reynolds, J. C. (1981). The essence of Algol. In de Bakker and van Vliet, editors, Algorithmic Languages, pages 345-372, Amsterdam. IFIP, North-Holland.

[Reynolds, 1998] Reynolds, J. C. (1998). Theories of programming languages. Cambridge University Press.

[Schmidt, 1994] Schmidt, D. A. (1994). The Structure of Typed Programming Languages. The MIT Press, Cambridge, Massachusetts.

[Schütte, 1967] Schütte, K. (1967). Proof theory. Addison Wesley.

[Stoy, 1977] Stoy, J. (1977). Denotational Semantics of Programming Languages: The Scott-Strachey Approach to Programming Language Theory. MIT.

[Talpin and Jouvelot, 1994] Talpin, J.-P. and Jouvelot, P. (1994). The type and effect discipline. Information and Computation, 111(2):245-296.

[Turner, 2004] Turner, D. A. (2004). Total functional programming. J. UCS, 10(7):751-768.

[Wadler, 1990] Wadler, P. (1990). Comprehending monads. In ACM Conference on Lisp and Functional Programming, pages 61-78, Nice, France.

[Wadler, 1998] Wadler, P. (1998). The marriage of effects and monads. In International Conference on Functional Programming, pages 63-74, Baltimore. ACM. 


\section{Table of contents}

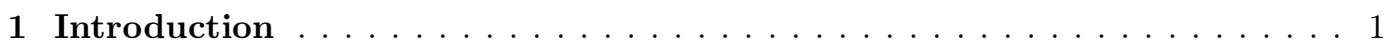

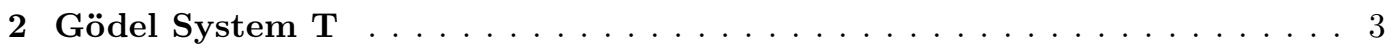

2.1 Example: the Ackermann function $\ldots \ldots \ldots \ldots \ldots \ldots$

3 The higher-order Loop language $\ldots \ldots \ldots \ldots \ldots \ldots \ldots$

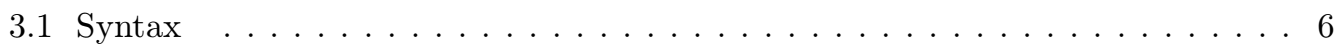

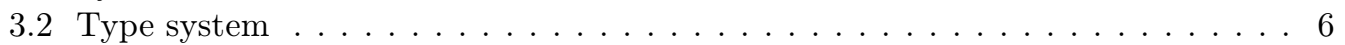

3.3 Natural semantics . . . . . . . . . . . . . . . . . . . 8

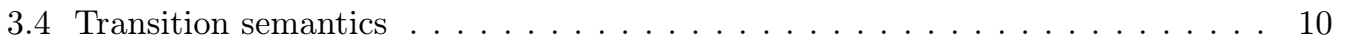

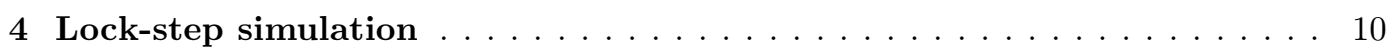

4.1 Translation . . . . . . . . . . . . . . . . . . . . . . . 10

4.2 Example: the Ackermann function . . . . . . . . . . . . . . . . . 13

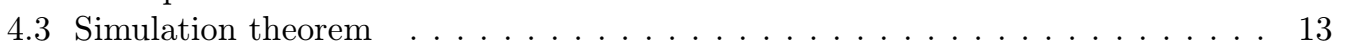

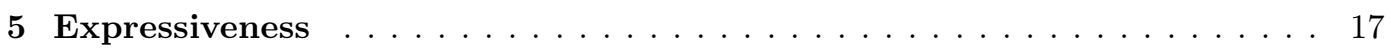

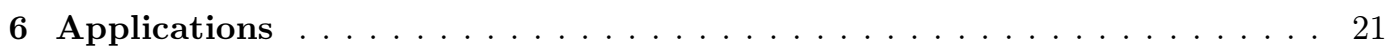

6.1 Characterizing elementary functions $\ldots \ldots \ldots \ldots \ldots \ldots \ldots \ldots \ldots$

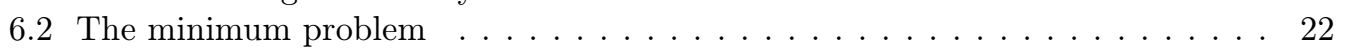

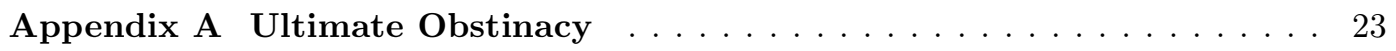

Appendix B Subject reduction for $\operatorname{Loop}^{\omega} \ldots \ldots \ldots \ldots \ldots \ldots$

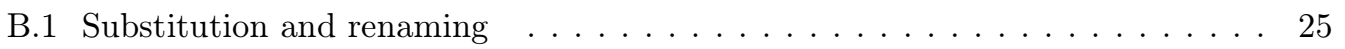

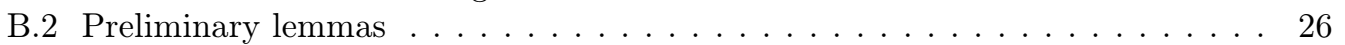

B.3 Proof of Theorem $3.18 \ldots \ldots \ldots \ldots \ldots \ldots \ldots$

Appendix C Translation ()$^{\star}$ is type-preserving $\ldots \ldots \ldots \ldots 28$

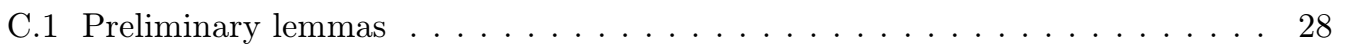

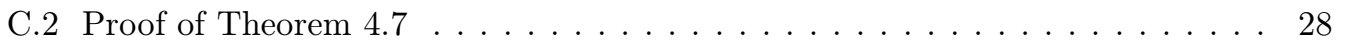

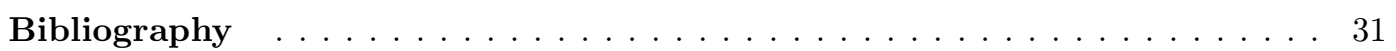

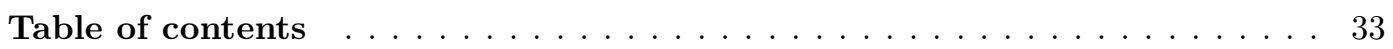

